\title{
Equilibrium fluctuation for an anharmonic chain with boundary conditions in the Euler scaling limit
}

\author{
Stefano Olla $\quad \mathrm{Lu} \mathrm{XU}$
}

口

\begin{abstract}
We study the evolution in equilibrium of the fluctuations for the conserved quantities of a chain of anharmonic oscillators in the hyperbolic space-time scaling. Boundary conditions are determined by applying a constant tension at one side, while the position of the other side is kept fixed. The Hamiltonian dynamics is perturbed by random terms conservative of such quantities. We prove that these fluctuations evolve macroscopically following the linearized Euler equations with the corresponding boundary conditions, even in some time scales larger than the hyperbolic one.
\end{abstract}

\section{Introduction}

The deduction of Euler equations for a compressible gas from the microscopic dynamics under a space-time scaling limit is one of the main problems in statistical mechanics 16. With a generic assumption of local equilibrium, Euler equations can be formally obtained in the limit, but a mathematical proof starting from deterministic Hamiltonian dynamics is still an open problem. The eventual appearance of shock waves complicates further the task, and in this case, it is expected the convergence to weak entropic solutions of Euler equations.

Some mathematical results have been obtained by perturbing the Hamiltonian dynamics by random terms that conserve only energy and momentum, in such a way that the dynamics has enough ergodicity to generate some form of local equilibrium (cf. [18, 6]). These results are obtained by relative entropy techniques and restricted to the smooth regime of the Euler equations. The noise introduced in these works are essentially random collisions between close particles and acts only on the velocities. Under such random perturbations, the only conserved quantities are those that evolve macroscopically with the Euler equations 11. Actually, random dynamics and local equilibrium are only tools to obtain the separation of scales between microscopic and macroscopic modes necessary in order to close the Euler equations. In the deterministic dynamics of harmonic oscillators with random masses (not ergodic), Anderson localization provides such separation of scales [5].

In this article we study the evolution of the fluctuations of the conserved quantities. When the system is in equilibrium at certain averaged values of the conserved quantities, these have Gaussian macroscopic fluctuations. The aim is to prove that these fluctuations, in the macroscopic space-time scaling limit, evolve deterministically following the linearized Euler equations. It turns out that this is more difficult than proving the hydrodynamic limit, as it requires the control of the space-time variance of the currents of the conserved quantities. More precisely it demands to prove that the currents are equivalent (in the norm introduced by the space-time variance) to linear functions of the conserved quantities. This step is usually called BoltzmannGibbs principle (cf. 7, 13]). This is the main part of the proof, and it forces us to consider elliptic type of stochastic perturbations, i.e., noise terms that act also on the positions, not only on the velocities, still maintaining the same conserved quantities.

July 2, 2019 
The system we consider is $N+1$ coupled anharmonic oscillators, similar to the one considered in [6]. For $i=0, \ldots, N$, the momentum (or velocity, since we set the masses equal to 1 ) of the particle $i$ is denoted by $p_{i} \in \mathbb{R}$, while $q_{i} \in \mathbb{R}$ denotes its position. Particle 0 is attached to some fixed point, thus $p_{0}=0, q_{0}=0$. Meanwhile, particle $N$ is pulled (or pushed) by a force $\tau \in \mathbb{R}$, which is constant in time.

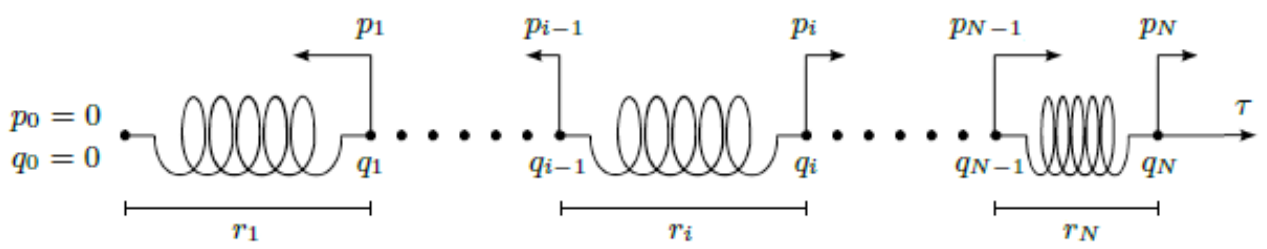

Each pair of consecutive particles $(i-1, i)$ is connected by a (nonlinear) spring with potential $V\left(q_{i}-q_{i-1}\right)$. We need to assume certain assumptions for the potential energy $V: \mathbb{R} \rightarrow \mathbb{R}$. The energy of the system is then given by

$$
H_{N}(\mathbf{p}, \mathbf{q})=\sum_{i=1}^{N}\left[\frac{p_{i}^{2}}{2}+V\left(q_{i}-q_{i-1}\right)\right] .
$$

Therefore, the inter-particle distances $\left\{r_{i}=q_{i}-q_{i-1} ; 1 \leq i \leq N\right\}$ are the essentially relevant variables. Notice that here $r_{i}$ can also assume negative values. Let $e_{i}=p_{i}^{2} / 2+V\left(r_{i}\right)$ be the energy assigned to $i$-th particle, then $H_{N}=\sum e_{i}$. The corresponding Hamiltonian dynamics locally conserves the sums of $p_{i}, r_{i}$ and $e_{i}$. By adding proper stochastic perturbations on the deterministic dynamics, we can make them the only conserved quantities.

Let $w_{i}=\left(p_{i}, r_{i}, e_{i}\right)$ be the vector of conserved quantities. The hydrodynamic limit is given by the convergence, for any continuous $G$ on $[0,1]$,

$$
\frac{1}{N} \sum_{i=1}^{N} w_{i}(N t) G\left(\frac{i}{N}\right) \underset{N \rightarrow \infty}{\longrightarrow} \int_{0}^{1} \mathfrak{w}(t, x) G(x) d x,
$$

where $\mathfrak{w}=(\mathfrak{p}, \mathfrak{r}, \mathfrak{e})$ solves the compressible Euler equations

$$
\partial_{t} \mathfrak{w}=\partial_{x} F(\mathfrak{w}), \quad F(\mathfrak{w})=(\boldsymbol{\tau}(\mathfrak{r}, \mathfrak{u}), \mathfrak{p}, \boldsymbol{\tau}(\mathfrak{r}, \mathfrak{u}) \mathfrak{p}), \quad \mathfrak{u}=\mathfrak{e}-\mathfrak{p}^{2} / 2
$$

with boundary conditions given by

$$
\mathfrak{p}(0, t)=0, \quad \boldsymbol{\tau}(\mathfrak{r}(1, t), \mathfrak{u}(1, t))=\tau,
$$

where $\boldsymbol{\tau}(r, e)$ is the tension function defined in (2.7) later. In the smooth regime of (1.1), this is proven by relative entropy techniques in [6].

We consider here the system in equilibrium, starting with the Gibbs measure

$$
\prod_{i=1}^{N} \exp \left\{\lambda \cdot\left(r_{i}, e_{i}\right)-\mathscr{G}(\lambda)\right\} d p_{i} d r_{i}
$$

for given $\lambda=(\beta \tau,-\beta) \in \mathbb{R} \times \mathbb{R}_{-}$, where $\mathscr{G}$ is the Gibbs potential given by

$$
\mathscr{G}(\lambda)=\ln \left(\int_{\mathbb{R}} \exp \{-\beta V(r)+\beta \tau r\} d r\right)+\frac{1}{2} \ln \left(\frac{2 \pi}{\beta}\right) .
$$


Denote by $E_{\lambda, N}$ the expectation with respect to the measure in 1.27 . Correspondingly, there are equilibrium values $0=E_{\lambda, N}\left[p_{i}\right], \bar{r}=E_{\lambda, N}\left[r_{i}\right], \bar{e}=E_{\lambda, N}\left[e_{i}\right]$ for the conserved quantities. The empirical distribution of the fluctuations of the conserved quantities is defined by

$$
\frac{1}{\sqrt{N}} \sum_{i=1}^{N}\left(\begin{array}{c}
p_{i}(N t) \\
r_{i}(N t)-\bar{r} \\
e_{i}(N t)-\bar{e}
\end{array}\right) \delta\left(x-\frac{i}{N}\right) .
$$

Formally, it is expected to converge to the solution $\tilde{\mathfrak{w}}=(\tilde{\mathfrak{p}}, \tilde{\mathfrak{r}}, \tilde{\mathfrak{e}})$ of

$$
\partial_{t} \tilde{\mathfrak{w}}=F^{\prime}(0, \bar{r}, \bar{e}) \partial_{x} \tilde{\mathfrak{w}},
$$

where $F^{\prime}(\bar{w})$ is the Jacobian matrix of $F$, with boundary conditions

$$
\tilde{\mathfrak{p}}(t, 0)=0,\left.\quad \frac{\partial \boldsymbol{\tau}}{\partial r}\right|_{(\bar{r}, \bar{e})} \tilde{\mathfrak{r}}(t, 1)+\left.\frac{\partial \boldsymbol{\tau}}{\partial e}\right|_{(\bar{r}, \bar{e})} \tilde{\mathfrak{e}}(t, 1)=0,
$$

and a proper Gaussian stationary initial distribution. Notice that $\tilde{\mathfrak{w}}(t)$ takes values as distributions on $[0,1]$, so $(1.4)$ with the boundary conditions (1.5) should be intended in the weak sense, as rigorously defined in Section 3

While the non-equilibrium hydrodynamic limit can be proven by adding a simple exchange of $p_{i}$ with $p_{i+1}$ at random independent times (cf. [6]), in order to prove (1.4) we need to add, for each bond $(i, i+1)$, a stochastic perturbation that exchanges $\left(p_{i}, p_{i+1}, r_{i}, r_{i+1}\right)$ in such way that $r_{i}+r_{i+1}, p_{i}+p_{i+1}, e_{i}+e_{i+1}$ are conserved. The corresponding microcanonical surface is a onedimensional circle, where we add a Wiener process. This stochastic perturbation corresponds to adding a symmetric second order differential operator $\mathcal{S}_{N}$ defined by $(2.2)$ that is elliptic on the corresponding microcanonical surfaces. The main part of the article is the proof of a lower bound of order $N^{-2}$ on the spectral gap of $\mathcal{S}_{N}$ that is independent of the values of the conserved quantities. This is an important ingredient for proving the Boltzmann-Gibbs linearization for the dynamics.

The present article contains the first result on equilibrium fluctuations for anharmonic chain of oscillators with multiple conserved quantities. Previous results concerned only linear dynamics or vanishing anharmonicity (eg. 4 for a system with two conserved quantities). Another novelty of the present article is the presence of non-linear boundary conditions (tension at the border), as previous results on equilibrium fluctuations concern systems with no boundary conditions, or linear in the conserved quantities.

The hyperbolic scale describes the time for the system to reach its mechanical equilibrium. Beyond that, it takes more time to reach the thermal equilibrium. It is a natural question to investigate the behaviour of the equilibrium fluctuations in larger time scales. In Theorem 3.3 we prove for our anharmonic system that the equilibrium fluctuations on the three conserved quantities continue to evolve deterministically according to the linearized Euler equations up to a time scale $N^{a} t$ with $a \in[1,6 / 5)$. For harmonic chain with two conserved quantities and no boundary conditions an analogous result can be found in [3]. Superdiffusion of energy fluctuations is conjectured in 21, and should appear for some $a \geq 3 / 2$. This has been proven rigorously for harmonic chains with conservative noise (cf. [12] for dynamics with 3 conserved quantities and [2] with two conserved quantities). Results in [12] extends also to the nonstationary superdiffusive evolution of the energy density, while the other two quantities evolve diffusively [15. See also the review [1] and the other articles in the same volume about the numerical evidence in non-linear dynamics. The extension of such superdiffusive results to the non-linear dynamics is one of the most challenging problem. Some results for vanishing anharmonicity can be found in [4].

We believe that such macroscopic behavior of the equilibrium fluctuations should be valid also for the deterministic (non-linear) dynamics, but even the case with a stochastic perturbation acting only on the velocities remains an open problem. 
Another important open problem concerns the evolution of fluctuations out of equilibrium. For system with one conserved quantity, like the asymmetric simple exclusion, in the context of the hyperbolic scaling this has been proven in [20].

\section{The microscopic model}

In this section we state the rigorous definition of the microscopic dynamics. Let $V$ be a convex, $C^{4}$-smooth function on $\mathbb{R}$ with quadratic growth:

$$
\inf _{r \in \mathbb{R}} V^{\prime \prime}(r)>0, \quad \sup _{r \in \mathbb{R}} V^{\prime \prime}(r)<\infty .
$$

Observe that (2.1) assures that $V(r)$ acquires its minimum at some unique point $r_{0} \in \mathbb{R}$. By replacing $V$ with $V_{*}=V\left(\cdot+r_{0}\right)-V\left(r_{0}\right)$, we can assume without loss of generality that $V \geq 0$, $V(0)=0$ and $V^{\prime}(0)=0$.

For $N \geq 1$, let $\Omega_{N}=\mathbb{R}^{2 N}$ be the configuration space. Its elements are denoted by

$$
\eta=(\mathbf{p}, \mathbf{r}) ; \quad \mathbf{p}=\left(p_{1}, \ldots, p_{N}\right), \mathbf{r}=\left(r_{1}, \ldots, r_{N}\right) .
$$

Fix $\tau \in \mathbb{R}, p_{0}=0$, and define first-order differential operators $\mathcal{X}_{i}$ acting on smooth functions on $\Omega_{N}$ by

$$
\begin{aligned}
& \mathcal{X}_{i}=\left(p_{i}-p_{i-1}\right) \frac{\partial}{\partial r_{i}}+\left(V^{\prime}\left(r_{i+1}\right)-V^{\prime}\left(r_{i}\right)\right) \frac{\partial}{\partial p_{i}}, \quad \text { for } 1 \leq i \leq N-1, \\
& \mathcal{X}_{N}=\left(p_{N}-p_{N-1}\right) \frac{\partial}{\partial r_{N}}+\left(\tau-V^{\prime}\left(r_{N}\right)\right) \frac{\partial}{\partial p_{N}}
\end{aligned}
$$

In addition, define $\mathcal{Y}_{i, i+1}$ for $1 \leq i \leq N-1$ as

$$
\mathcal{Y}_{i, i+1}=\left(p_{i+1}-p_{i}\right)\left(\frac{\partial}{\partial r_{i+1}}-\frac{\partial}{\partial r_{i}}\right)-\left(V^{\prime}\left(r_{i+1}\right)-V^{\prime}\left(r_{i}\right)\right)\left(\frac{\partial}{\partial p_{i+1}}-\frac{\partial}{\partial p_{i}}\right) \text {. }
$$

For any $\gamma>0$, the generator $\mathcal{L}_{N}$ is given by

$$
\mathcal{L}_{N}=\mathcal{A}_{N}+\gamma \mathcal{S}_{N}, \quad \mathcal{A}_{N}=\sum_{i=1}^{N} \mathcal{X}_{i}, \quad \mathcal{S}_{N}=\frac{1}{2} \sum_{i=1}^{N-1} \mathcal{Y}_{i, i+1}^{2}
$$

The Liouville operator $\mathcal{A}_{N}$ generates the Hamiltonian system introduced in Section 11, while each $\mathcal{Y}_{i, i+1}$ generates a continuous stochastic perturbation on $\left(p_{i}, p_{i+1}, r_{i}, r_{i+1}\right)$, preserving the amounts of $p_{i}+p_{i+1}, r_{i}+r_{i+1}$ and $e_{i}+e_{i+1}$. This choice of noises assures that $p_{i}, r_{i}$ and $e_{i}$ are the only locally conserved quantities.

Denote by $\pi_{\lambda, N}$ the Gibbs measure in 1.2 . The class of bounded, smooth functions on $\Omega_{N}$ forms a core of $\mathcal{A}_{N}$ and $\mathcal{S}_{N}$ in $L^{2}\left(\pi_{\lambda, N}\right)$, and for such $f$ and $g$,

$$
E_{\lambda, N}\left[\left(\mathcal{A}_{N} f\right) g\right]=-E_{\lambda, N}\left[f\left(\mathcal{A}_{N} g\right)\right], \quad E_{\lambda, N}\left[\left(\mathcal{S}_{N} f\right) g\right]=E_{\lambda, N}\left[f\left(\mathcal{S}_{N} g\right)\right] .
$$

In particular, $\pi_{\lambda, N}$ is stationary with respect to $\mathcal{L}_{N}$. Moreover,

$$
E_{\lambda, N}\left[f\left(-\mathcal{L}_{N} f\right)\right]=\gamma E_{\lambda, N}\left[f\left(-\mathcal{S}_{N} f\right)\right]=\frac{\gamma}{2} \sum_{i=1}^{N} E_{\lambda, N}\left[\left(\mathcal{Y}_{i, i+1} f\right)^{2}\right]
$$

Denote by $\bar{w}=E_{\lambda, N}\left[w_{i}\right]$, then $\bar{w}=(0, \bar{r}(\lambda), \bar{e}(\lambda))$, where

$$
(\bar{r}(\lambda), \bar{e}(\lambda))=\nabla_{\lambda} \mathscr{G}(\lambda)=\left(\frac{1}{\beta} \frac{\partial}{\partial \tau}, \frac{\tau}{\beta} \frac{\partial}{\partial \tau}-\frac{\partial}{\partial \beta}\right) \mathscr{G}
$$


where $\mathscr{G}(\lambda)$ is defined in 1.3 . It is also worth noticing that the tension in equilibrium is $E_{\lambda, N}\left[V^{\prime}\left(r_{i}\right)\right]=\tau$. Furthermore, the covariance matrix $\Sigma=\Sigma(\lambda)$ of $w_{i}$ under $\pi_{\lambda, N}$ is given by

$$
\Sigma=E_{\lambda, N}\left[\left(w_{i}-\bar{w}\right) \otimes\left(w_{i}-\bar{w}\right)\right]=\left[\begin{array}{c|cc}
\beta^{-1} & 0 & 0 \\
\hline 0 & \mathscr{G}^{\prime \prime}(\lambda)
\end{array}\right],
$$

where $\mathscr{G}^{\prime \prime}(\lambda)$ stands for the Hessian matrix of $\mathscr{G}$ calculated in $\lambda$.

Define the thermodynamic entropy $\mathscr{S}$ for $r \in \mathbb{R}$ and $e>0$ by

$$
\mathscr{S}(r, e)=-\mathscr{G}^{*}(r, e), \quad \mathscr{G}^{*}=\sup _{\lambda \in \mathbb{R} \times \mathbb{R}_{-}}\{\lambda \cdot(r, e)-\mathscr{G}(\lambda)\} .
$$

Under our assumptions, $\mathscr{G}$ is strictly convex and so is its Legendre transform $\mathscr{G}^{*}$. Hence, $\mathscr{S}$ is strictly concave. By the general theories in Legendre transform,

$$
\boldsymbol{\lambda}(r, e)=\nabla_{r, e} \mathscr{G}^{*}(r, e)=-\nabla_{r, e} \mathscr{S}(r, e) \in \mathbb{R} \times \mathbb{R}_{-}
$$

gives the inverse of $\lambda \rightarrow \nabla_{\lambda} \mathscr{G}(\lambda)$. In view of (2.3),

$$
\mathscr{G}^{\prime \prime}(\lambda) \mathscr{S}^{\prime \prime}(\bar{r}(\lambda), \bar{e}(\lambda))=\mathscr{G}^{\prime \prime}(\boldsymbol{\lambda}(r, e)) \mathscr{S}^{\prime \prime}(r, e)=-I_{2 \times 2} .
$$

For convenience, we denote $\boldsymbol{\lambda}=(\boldsymbol{\beta} \boldsymbol{\tau},-\boldsymbol{\beta})$, where

$$
\boldsymbol{\beta}(r, e)=\partial_{e} \mathscr{S}(r, e), \quad \boldsymbol{\tau}(r, e)=-\frac{\partial_{r} \mathscr{S}(r, e)}{\partial_{e} \mathscr{S}(r, e)} .
$$

By $(2.5), \boldsymbol{\beta}(r, e)$ is always positive, and

$$
\begin{aligned}
\frac{\partial \boldsymbol{\tau}}{\partial r}+\boldsymbol{\tau} \frac{\partial \boldsymbol{\tau}}{\partial e} & =\frac{1}{\boldsymbol{\beta}}\left(-\frac{\partial^{2} \mathscr{S}}{(\partial r)^{2}}+\frac{1}{\boldsymbol{\beta}} \frac{\partial \boldsymbol{\beta}}{\partial r} \frac{\partial \mathscr{S}}{\partial r}\right)-\frac{1}{\boldsymbol{\beta}^{2}} \frac{\partial \mathscr{S}}{\partial r}\left(-\frac{\partial^{2} \mathscr{S}}{\partial r \partial e}+\frac{1}{\boldsymbol{\beta}} \frac{\partial \boldsymbol{\beta}}{\partial e} \frac{\partial \mathscr{S}}{\partial r}\right) \\
& =-\frac{1}{\boldsymbol{\beta}^{3}}\left(\boldsymbol{\beta}^{2} \frac{\partial^{2} \mathscr{S}}{(\partial r)^{2}}-\boldsymbol{\beta} \frac{\partial^{2} \mathscr{S}}{\partial r \partial e} \frac{\partial \mathscr{S}}{\partial r}-\boldsymbol{\beta} \frac{\partial^{2} \mathscr{S}}{\partial r \partial e} \frac{\partial \mathscr{S}}{\partial r}+\frac{\partial^{2} \mathscr{S}}{\partial e^{2}}\left[\frac{\partial \mathscr{S}}{\partial r}\right]^{2}\right) \\
& =\frac{1}{\boldsymbol{\beta}^{3}}\left(\frac{\partial \mathscr{S}}{\partial e},-\frac{\partial \mathscr{S}}{\partial r}\right) \cdot(-\mathscr{S})^{\prime \prime}\left(\frac{\partial \mathscr{S}}{\partial e},-\frac{\partial \mathscr{S}}{\partial r}\right) .
\end{aligned}
$$

Since $\mathscr{S}$ is strictly concave, one can conclude that

$$
\frac{\partial \boldsymbol{\tau}}{\partial r}+\boldsymbol{\tau} \frac{\partial \boldsymbol{\tau}}{\partial e}>0
$$

For each $N \geq 1$, denote by $\left\{\eta_{t} \in \Omega_{N} ; t \geq 0\right\}$ the Markov process generated by $N \mathcal{L}_{N}$. Observe that $\eta_{t}=(\mathbf{p}(t), \mathbf{r}(t))$ can be equivalently expressed by the solution to the following system of stochastic differential equations:

$$
\left\{\begin{aligned}
d p_{1}(t) & =N \nabla_{N} V^{\prime}\left(r_{1}\right) d t+d J_{1}^{p}, \\
d p_{i}(t) & =N \nabla_{N} V^{\prime}\left(r_{i}\right) d t-\nabla_{N}^{*} d J_{i}^{p}, \quad \text { for } 2 \leq i \leq N-1, \\
d p_{N}(t) & =N\left[\tau-V^{\prime}\left(r_{N}\right)\right] d t-d J_{N-1}^{p}, \\
d r_{1}(t) & =N p_{1} d t+d J_{1}^{r}, \\
d r_{i}(t) & =N \nabla_{N} p_{i-1} d t-\nabla_{N}^{*} d J_{i}^{r}, \quad \text { for } 2 \leq i \leq N-1, \\
d r_{N}(t) & =N \nabla_{N} p_{N-1} d t-d J_{N-1}^{r},
\end{aligned}\right.
$$

where for any sequence $\left\{f_{i}\right\}, \nabla_{N} f_{i}=f_{i+1}-f_{i}, \nabla_{N}^{*} f_{i}=f_{i-1}-f_{i}$,

$$
\begin{aligned}
& d J_{i}^{p}=\frac{\gamma N}{2}\left[V^{\prime \prime}\left(r_{i+1}\right)+V^{\prime \prime}\left(r_{i}\right)\right] \nabla_{N} p_{i} d t+\sqrt{\gamma N}\left(\nabla_{N} V^{\prime}\left(r_{i}\right)\right) d B_{t}^{i}, \\
& d J_{i}^{r}=\gamma N \nabla_{N} V^{\prime}\left(r_{i}\right) d t-\sqrt{\gamma N}\left(\nabla_{N} p_{i}\right) d B_{t}^{i},
\end{aligned}
$$


and $\left\{B^{i} ; i \geq 1\right\}$ is an infinite system of independent, standard Brownian motions. Let $\mathbb{P}_{\lambda, N}$ be the probability measure on the path space $C\left([0, \infty), \Omega_{N}\right)$ induced by $(2.9)$ and initial condition $\pi_{\lambda, N}$. The corresponding expectation is denoted by $\mathbb{E}_{\lambda, N}$.

We are interested in the evolution of the fluctuations of the balanced quantities of $\left\{\eta_{t}\right\}$ in macroscopic time. For a smooth function $H:[0,1] \rightarrow \mathbb{R}^{3}$, define the empirical distribution of conserved quantities fluctuation field on $H$ as

$$
Y_{N}(t, H)=\frac{1}{\sqrt{N}} \sum_{i=1}^{N} H\left(\frac{i}{N}\right) \cdot\left(w_{i}\left(\eta_{t}\right)-\bar{w}\right), \quad \forall t \geq 0,
$$

Notice that we consider in 2.10 the hyperbolic scaling, where the space and time variables are rescaled by the same order of $N$.

We close this section with some useful notations. Throughout this article, $|\cdot|$ and $\cdot$ always refer to the standard Euclidean norm and inner product in $\mathbb{R}^{d}$. Let $\mathscr{H}$ be the space of threedimensional functions $f=\left(f_{1}, f_{2}, f_{3}\right)$ on $[0,1]$, where each $f_{i}$ is square integrable. The scalar product and norm on $\mathscr{H}$ are given by

$$
\langle f, g\rangle=\int_{0}^{1} f(x) \cdot g(x) d x, \quad\|f\|^{2}=\int_{0}^{1}|f(x)|^{2} d x .
$$

Then $\mathscr{H}$ is a Hilbert space, and denote by $\mathscr{H}^{\prime}$ its dual space, consisting of all bounded linear functionals on $\mathscr{H}$. Note that the definition in 2.10 satisfies that:

$$
\mathbb{E}_{\lambda, N}\left[Y_{N}^{2}(t, H)\right] \leq|\operatorname{Tr} \Sigma(\lambda)| \cdot \frac{1}{N} \sum_{i=1}^{N}\left|H\left(\frac{i}{N}\right)\right|^{2}
$$

Thus, one can easily extend the definition of $Y_{N}(t, H)$ to all $H \in \mathscr{H}$. For all $N \geq 1, t \geq 0$ and $H \in \mathscr{H}, Y_{N}(t, H) \in L^{2}\left(\Omega_{N} ; \pi_{\lambda, N}\right)$.

\section{Euler system with boundary conditions}

In this section we state the precise definition of the solution to 1.4 , 1.5 with proper random distribution-valued initial condition. The equation (1.4) can be written explicitly as

$$
\partial_{t} \tilde{\mathfrak{p}}=\tau_{r} \partial_{x} \tilde{\mathfrak{r}}+\tau_{e} \partial_{x} \tilde{\mathfrak{e}}, \quad \partial_{t} \tilde{\mathfrak{r}}=\partial_{x} \tilde{\mathfrak{p}}, \quad \partial_{t} \tilde{\mathfrak{e}}=\tau \partial_{x} \tilde{\mathfrak{p}},
$$

where $\left(\tau_{r}, \tau_{e}\right)$ are constants given by

$$
\tau_{r}(\lambda)=\frac{\partial}{\partial r} \boldsymbol{\tau}(\bar{r}(\lambda), \bar{e}(\lambda)), \quad \tau_{e}(\lambda)=\frac{\partial}{\partial e} \boldsymbol{\tau}(\bar{r}(\lambda), \bar{e}(\lambda))
$$

Recall that $(\boldsymbol{\beta}, \boldsymbol{\tau})(\bar{r}(\lambda), \bar{e}(\lambda))=(\beta, \tau)$ are constants, and by $(2.7), \partial_{r} \mathscr{S}=-\boldsymbol{\beta} \boldsymbol{\tau}, \partial_{e} \mathscr{S}=\boldsymbol{\beta}$. Formally define the linear transformation

$$
\tilde{\tau}=\tau_{r} \tilde{\mathfrak{r}}+\tau_{e} \tilde{\mathfrak{e}}, \quad \tilde{S}=-\beta \tau \tilde{\mathfrak{r}}+\beta \tilde{\mathfrak{e}} .
$$

The new coordinates $\tilde{\tau}, \tilde{S}$ can be viewed as the fluctuation field of tension and thermodynamic entropy, respectively. From $(1.4),(\tilde{\mathfrak{p}}, \tilde{\tau}, \tilde{S})$ evolves with the equation

$$
\partial_{t} \tilde{\mathfrak{p}}=\partial_{x} \tilde{\tau}, \quad \partial_{t} \tilde{\tau}=c^{2} \partial_{x} \tilde{\mathfrak{p}}, \quad \partial_{t} \tilde{S}=0
$$

where the constant $c>0$ is the speed of sound given by

$$
c^{2}=\tau_{r}+\tau \tau_{e}>0
$$


cf. 2.8) and [21, (3.10)]. This transformation also decouples the boundary conditions:

$$
\tilde{\mathfrak{p}}(t, 0)=0, \quad \tilde{\tau}(t, 1)=0 .
$$

It turns to be clear that $(\tilde{\mathfrak{p}}, \tilde{\tau})$ are two coupled sound modes with mixed boundaries, while $\tilde{S}$ is independent of $(\tilde{\mathfrak{p}}, \tilde{\tau})$ and does not evolve in time. Suppose that the initial data is smooth and satisfies the boundary conditions, one can easily obtain the smooth solution $\tilde{\mathfrak{w}}=\tilde{\mathfrak{w}}(t, x)$ to (1.4), (1.5) by applying the inverse transformation.

Since $\tilde{\mathfrak{w}}(0)$ is a Gaussian random filed, to present the idea above rigorously, we have to consider the weak solution. Define a subspace $\mathscr{C}(\lambda)$ of $\mathscr{H}$ by

$$
\mathscr{C}(\lambda)=\left\{g=\left(g_{1}, g_{2}, g_{3}\right) \mid g_{i} \in C^{1}([0,1]), g_{1}(0)=0, \tau_{r} g_{2}(1)+\tau_{e} g_{3}(1)=0\right\} .
$$

Define the first-order differential operator $L$ on $\mathscr{C}(\lambda)$ by

$$
L=B\left(\frac{d}{d x}\right), \quad \text { where } B=F^{\prime}(\bar{w})=\left[\begin{array}{ccc}
0 & \tau_{r} & \tau_{e} \\
1 & 0 & 0 \\
\tau & 0 & 0
\end{array}\right] .
$$

Observe that $B$ has three real eigenvalues $\{0, \pm c\}$, thus generates a hyperbolic system. With some abuse of notations, denote the closure of $L$ on $\mathscr{H}$ still by $L$. For $i=1,2$, let $\left\{\mu_{i, n} ; n \geq 0\right\}$ be two Fourier bases of $L^{2}([0,1])$ given by

$$
\mu_{1, n}(x)=\sqrt{2} \sin \left(\theta_{n} x\right), \quad \mu_{2, n}(x)=\sqrt{2} \cos \left(\theta_{n} x\right), \quad \theta_{n}=\frac{(2 n+1) \pi}{2} .
$$

Notice that $\mu_{1, n}(0)=\mu_{2, n}(1)=0$, in accordance with the boundary conditions in 3.3 . For $k \geq 1$, define the Sobolev spaces

$$
H_{k}=\left\{f=\left(f_{1}, f_{2}\right) \mid \sum_{i=1}^{2} \sum_{n=0}^{\infty} \theta_{n}^{2 k}\left(\int_{0}^{1} f_{i}(x) \mu_{i, n}(x) d x\right)^{2}<\infty\right\} .
$$

Then $\operatorname{dom}(L)=\left\{\left(g_{1}, \tau_{r} g_{2}+\tau_{e} g_{3}\right) \in H_{1}\right\}$. To identify the adjoint $L^{*}$ of $L$, observe that for any $g \in \mathscr{C}(\lambda)$ and $h \in \mathscr{H}$,

$$
\langle L g, h\rangle=\int_{0}^{1} g_{1}^{\prime}\left(h_{2}+\tau h_{3}\right)+\left(\tau_{r} g_{2}^{\prime}+\tau_{e} g_{3}^{\prime}\right) h_{1} d x .
$$

Therefore, $\operatorname{dom}\left(L^{*}\right)=\left\{\left(h_{1}, h_{2}+\tau h_{3}\right) \in H_{1}\right\}$. In particular,

$$
\mathscr{C}_{*}(\tau)=\left\{h=\left(h_{1}, h_{2}, h_{3}\right) \mid h_{i} \in C^{1}([0,1]), h_{1}(0)=0, h_{2}(1)+\tau h_{3}(1)=0\right\}
$$

is a core of $L^{*}$ and $L^{*} h=-B^{T} h^{\prime}$ for $h \in \mathscr{C}_{*}(\tau)$. Notice that $\mathscr{C}_{*}(\tau)$ depends only on $\tau$, while $\mathscr{C}(\lambda)$ depends on both $\beta$ and $\tau$.

Now we can state the definition of 1.4 and 1.5 precisely. Let $\{\tilde{\mathfrak{w}}(t)=\tilde{\mathfrak{w}}(t, \cdot) ; t \geq 0\}$ be a stochastic process taking values in $\mathscr{H}^{\prime}$, such that for all $h \in \mathscr{C}_{*}(\tau)$,

$$
\tilde{\mathfrak{w}}(t, h)-\tilde{\mathfrak{w}}(0, h)=\int_{0}^{t} \tilde{\mathfrak{w}}\left(s, L^{*} h\right) d s, \quad \forall t>0,
$$

and $\tilde{\mathfrak{w}}(0)$ is a Gaussian variable such that for $h, g \in \mathscr{H}$,

$$
E[\tilde{\mathfrak{w}}(0, h)]=0, \quad E[\tilde{\mathfrak{w}}(0, h) \tilde{\mathfrak{w}}(0, g)]=\langle h, \Sigma g\rangle,
$$

where $\Sigma$ is the covariance matrix defined in 2.4 . 
To see the existence and uniqueness of $\tilde{\mathfrak{w}}(t)$, consider the weak form of equation 3.2 : for $f=\left(f_{1}, f_{2}, f_{3}\right), f_{i} \in C^{1}([0,1]), f_{1}(0)=f_{2}(1)=0$,

$$
\tilde{\mathfrak{u}}(t, f)-\tilde{\mathfrak{u}}(0, f)+\int_{0}^{t} \tilde{\mathfrak{u}}\left(s, A^{T} f^{\prime}\right) d s=0, \quad A=\left[\begin{array}{ccc}
0 & 1 & 0 \\
c^{2} & 0 & 0 \\
0 & 0 & 0
\end{array}\right],
$$

and $\tilde{\mathfrak{u}}(0)$ is a centered Gaussian variable with covariance

$$
E\left[(\tilde{\mathfrak{u}}(0, f))^{2}\right]=\langle f, Q f\rangle, \quad Q=\operatorname{diag}\left(\beta^{-1}, \beta^{-1} c^{2}, \beta^{2} \partial_{\beta}^{2} \mathscr{G}\right) .
$$

Suppose that $\left\{\boldsymbol{\mu}_{i, n}, \boldsymbol{\nu}_{i, n} ; n \geq 0, i=1,2\right\}$ is the three-dimensional Fourier bases given by $\boldsymbol{\mu}_{1, n}=$ $\left(\mu_{1, n}, 0,0\right), \boldsymbol{\mu}_{2, n}=\left(0, \mu_{2, n}, 0\right)$, and ${ }^{1}$

$$
\boldsymbol{\nu}_{1, n}(x)=\sqrt{2}\left(0,0, \sin \left(\kappa_{n} x\right)\right), \quad \boldsymbol{\nu}_{2, n}(x)=\sqrt{2}\left(0,0, \cos \left(\kappa_{n} x\right)\right), \quad \kappa_{n}=2 n \pi .
$$

The solution $\tilde{\mathfrak{u}}(t)$ is a stationary Gaussian process, satisfying that

$$
\begin{aligned}
\tilde{\mathfrak{u}}\left(t, \boldsymbol{\mu}_{1, n}\right) & =\frac{1}{\sqrt{\beta}}\left(X_{1, n} \cos \left(c \theta_{n} t\right)+X_{2, n} \sin \left(c \theta_{n} t\right)\right), \\
\tilde{\mathfrak{u}}\left(t, \boldsymbol{\mu}_{2, n}\right) & =\frac{c}{\sqrt{\beta}}\left(X_{1, n} \sin \left(c \theta_{n} t\right)-X_{2, n} \cos \left(c \theta_{n} t\right)\right), \\
\tilde{\mathfrak{u}}\left(t, \boldsymbol{\nu}_{i, n}\right) & =\beta \sqrt{\partial_{\beta}^{2} \mathscr{G}(\beta, \tau)} Y_{i, n},
\end{aligned}
$$

where $\left\{X_{i, n}, Y_{i, n} ; n \geq 0, i=1,2\right\}$ is an independent system of standard Gaussian random variables. The sample paths $\tilde{\mathfrak{u}}(\cdot) \in C\left([0, T] ; \mathscr{H}_{-1}\right)$ a.s., where

$$
\mathscr{H}_{-k}=\left\{\tilde{\mathfrak{u}} \mid \sum_{i=1}^{2} \sum_{n=0}^{\infty}\left\{\theta_{n}^{-2 k} \tilde{\mathfrak{u}}^{2}\left(\boldsymbol{\mu}_{i, n}\right)+\kappa_{n}^{-2 k} \tilde{\mathfrak{u}}^{2}\left(\boldsymbol{\nu}_{i, n}\right)\right\}<\infty\right\} .
$$

For each $h \in \mathscr{C}_{*}(\tau)$, define $\tilde{\mathfrak{w}}(t, h)$ by

$$
\tilde{\mathfrak{w}}(t, h)=\tilde{\mathfrak{u}}\left(t, R^{-1} h\right), \quad R=R(\lambda)=\left[\begin{array}{ccc}
1 & 0 & 0 \\
0 & \tau_{r} & -\beta \tau \\
0 & \tau_{e} & \beta
\end{array}\right] .
$$

Observing that $A^{T} R^{-1}=R^{-1} B^{T}$, and $f_{1}(0)=f_{2}(1)=0$ for $f \in R^{-1}\left[\mathscr{C}_{*}(\tau)\right]$,

$$
\tilde{\mathfrak{w}}(t, h)-\tilde{\mathfrak{w}}(0, h)=-\int_{0}^{t} \tilde{\mathfrak{u}}\left(s, A^{T} R^{-1} h^{\prime}\right) d s=-\int_{0}^{t} \tilde{\mathfrak{w}}\left(s, B^{T} h^{\prime}\right) d s,
$$

and 3.5 is fulfilled. On the other hand, from 2.7) and (3.1),

$$
\left(\begin{array}{c}
\tau_{r} \\
\tau_{e}
\end{array}\right)=-\left.\frac{1}{\beta}\left(\begin{array}{c}
\partial_{r}^{2} \mathscr{S} \\
\partial_{r} \partial_{e} \mathscr{S}
\end{array}\right)\right|_{(\bar{r}, \bar{e})}-\left.\frac{\tau}{\beta}\left(\begin{array}{c}
\partial_{r} \partial_{e} \mathscr{S} \\
\partial_{e}^{2} \mathscr{S}
\end{array}\right)\right|_{(\bar{r}, \bar{e})} .
$$

Combining this with 2.6), one obtains that

$$
\mathscr{G}^{\prime \prime}(\lambda)\left(\begin{array}{c}
\tau_{r} \\
\tau_{e}
\end{array}\right)=\left(\begin{array}{c}
\beta^{-1} \\
\beta^{-1} \tau
\end{array}\right)
$$

By this and some direct calculations,

$$
R^{T} \Sigma R=\operatorname{diag}\left(\beta^{-1}, \beta^{-1} c^{2}, \beta^{2} \partial_{\beta}^{2} \mathscr{G}\right)=Q,
$$

\footnotetext{
${ }^{1} \boldsymbol{\nu}_{i, n}$ are chosen arbitrarily, since this coordinate is a constant Gaussian random filed.
} 
therefore (3.6) also holds. In consequence, $\{\tilde{\mathfrak{w}}(t) ; t \in[0, T]\}$ uniquely exists in the path space $C\left([0, T] ; \mathscr{H}_{-k}(\lambda)\right)$ for $k \geq 1$, where

$$
\mathscr{H}_{-k}(\lambda)=\left\{\tilde{\mathfrak{w}} \mid\|\tilde{\mathfrak{w}}\|_{-k}^{2}=\sum_{i=1}^{2} \sum_{n=0}^{\infty}\left\{\theta_{n}^{-2 k} \tilde{\mathfrak{w}}^{2}\left(R \boldsymbol{\mu}_{i, n}\right)+\kappa_{n}^{-2 k} \tilde{\mathfrak{w}}^{2}\left(R \boldsymbol{\nu}_{i, n}\right)\right\}<\infty\right\},
$$

with three-dimensional Fourier bases $\boldsymbol{\mu}_{i, n}$ and $\boldsymbol{\nu}_{i, n}$ given in (3.4) and (3.8), and the Gaussian distribution determined by (3.6) is stationary for $\tilde{\mathfrak{w}}(t)$.

For $T>0$ and $k>5 / 2$, denote by $\mathbb{Q}_{N}$ the distribution of $\left\{Y_{N}(t) ; t \in[0, T]\right.$ on the path space $C\left([0, T], \mathscr{H}_{-k}(\lambda)\right)$ induced by $\mathbb{P}_{\lambda, N}$. Denote by $\mathbb{Q}$ the distribution of $\{\tilde{\mathfrak{w}}(t) ; t \in[0, T]\}$ defined above. Our first result is stated as below.

Theorem 3.1. Assume (2.1), then the sequence of probability measures $\left\{\mathbb{Q}_{N}\right\}$ converges weakly, as $N \rightarrow \infty$, to the probability measure $\mathbb{Q}$.

Remark 3.2. The condition $k>5 / 2$ is necessary only for the tightness in Section 7 .

Indeed, by the tightness of $\left\{\mathbb{Q}_{N}\right\}$ in Section 7 , we can pick an arbitrary limit point of $\mathbb{Q}_{N}$. Denote it by $\mathbb{Q}$ and let $\{Y(t)\}$ be a process subject to $\mathbb{Q}$. From classical central limit theorem, the distribution of $Y(0)$ satisfies $(3.6)$. By virtue of the uniqueness of the solution, to prove Theorem 3.1 it suffices to verify $(3.5)$, or equivalently,

$$
\left|Y_{N}(t, H(t, \cdot))-Y_{N}(0, h)\right| \rightarrow 0 \quad \text { in probability, }
$$

where $H(t, x)$ solves the backward Euler system:

$$
\partial_{t} H(t, x)+L^{*} H(t, x)=0, \quad H(0, \cdot)=h,
$$

for smooth initial data $h \in \mathscr{C}_{*}(\tau)$, with the following additional compatibility conditions also assumed at the space-time edges:

$$
\begin{array}{ll}
\lim _{x \rightarrow 0^{+}} \partial_{x} H_{1}(0, x)=0, & \lim _{x \rightarrow 1^{-}} \partial_{x}\left(H_{2}(0, x)+\tau H_{3}(0, x)\right)=0, \\
\lim _{t \rightarrow 0^{+}} \partial_{t}^{2} H_{1}(t, 0)=0, & \lim _{t \rightarrow 0^{+}} \partial_{t}^{2}\left(H_{2}(t, 1)+\tau H_{3}(t, 1)\right)=0 .
\end{array}
$$

Note that (3.11) assures that $H(t, \cdot) \in \mathscr{C}_{*}(\tau)$ is differentiable in $x$ up to the second order, and there exists a finite constant $C$ such that

$$
|H(t, x)| \leq C, \quad\left|\partial_{x} H(t, x)\right| \leq C, \quad\left|\partial_{x}^{2} H(t, x)\right| \leq C
$$

for any $t \geq 0$ and $x \in[0,1]$. As a further result of Theorem 3.1. we are able to prove that the fluctuation field keeps evolving with the linearized system for time scales beyond hyperbolic, under some additional assumptions.

Theorem 3.3. Assume 2.1]. There exists some universal $\delta>0$, such that if

$$
\sup _{r \in \mathbb{R}} V^{\prime \prime}(r)<(1+\delta) \inf _{r \in \mathbb{R}} V^{\prime \prime}(r),
$$

then for any $\alpha<1 / 5, T>0$ and $\epsilon>0$,

$$
\lim _{N \rightarrow \infty} \mathbb{P}_{\lambda, N}\left\{\exists t \in[0, T],\left|Y_{N}\left(N^{\alpha} t, H\left(N^{\alpha} t\right)\right)-Y_{N}(0, H(0))\right|>\epsilon\right\}=0,
$$

where $H(t)=H(t, x)$ solves the backward equation 3.10 and (3.11).

Remark 3.4. Theorem 3.3 shows that the fluctuation of thermodynamic entropy $\tilde{S}$ keeps stationary for any time scales $N^{a} t$ with $a<6 / 5$. It is expected that $\tilde{S}$ would evolve under some superdiffusive scaling $a<2$ following a fractional heat equation. 
Remark 3.5. Let $\mathbb{T}_{N}=\mathbb{Z} /(N \mathbb{Z})$ be the lattice torus with length $N$. One can also put the chain on $\mathbb{T}_{N}$ by applying the periodic boundary condition $\left(p_{0}, r_{0}\right)=\left(p_{N}, r_{N}\right)$ instead of the ones introduced in Section 1. Then, the equilibrium Gibbs measures become

$$
\pi_{\beta, \bar{p}, \tau}^{N}=\prod_{i \in \mathbb{T}_{N}} \exp \left\{\tilde{\lambda} \cdot w_{i}-\tilde{\mathscr{G}}(\tilde{\lambda})\right\} d p_{i} d r_{i}
$$

for given $\tilde{\lambda}=(\beta \bar{p}, \beta \tau,-\beta) \in \mathbb{R}^{2} \times \mathbb{R}_{-}$, where $\bar{p} \in \mathbb{R}$ denotes the momenta in equilibrium. For $(p, r) \in \mathbb{R}^{2}$ and $e \geq p^{2} / 2+V(r)$, we can define the internal energy $U=e-p^{2} / 2$, then the thermodynamic entropy and tension function are given by $\mathscr{S}(r, U)$ and $\boldsymbol{\tau}(r, U)$.

Start the dynamics from some equilibrium state $\pi_{\beta, \bar{p}, \tau}^{N}$. Let $\mathbb{T}=[0,1)$ stand for the onedimensional torus. For a bounded smooth function $H: \mathbb{T} \rightarrow \mathbb{R}$, the equilibrium fluctuation field is given by

$$
Y_{N}(t, H)=\frac{1}{\sqrt{N}} \sum_{i=1}^{N} H\left(\frac{i}{N}\right) \cdot\left(\begin{array}{c}
p_{i}(N t)-\bar{p} \\
r_{i}(N t)-\bar{r} \\
e_{i}(N t)-\bar{e}
\end{array}\right)
$$

With similar argument used to prove Theorem 3.1, we can show that $Y_{N}(t, H) \rightarrow \tilde{\mathfrak{w}}(t, H)$. Here $\tilde{\mathfrak{w}}(t, \cdot)$ solves the following linearized Euler system on torus:

$$
\partial_{t} \tilde{\mathfrak{w}}(t, x)=\left[\begin{array}{ccc}
-\bar{p} \tau_{u} & \tau_{r} & \tau_{u} \\
1 & 0 & 0 \\
\tau-\bar{p}^{2} \tau_{u} & \bar{p} \tau_{r} & \bar{p} \tau_{u}
\end{array}\right] \partial_{x} \tilde{\mathfrak{w}}(t, x)
$$

where the linear coefficients are given by

$$
\left(\tau_{r}, \tau_{u}\right)=\left(\partial_{r}, \partial_{u}\right) \boldsymbol{\tau}\left(\bar{r}, \bar{e}-\frac{\bar{p}^{2}}{2}\right)
$$

Similar to 3.2 , we have $\tilde{\mathfrak{p}}$ and $\tilde{\tau}=-\bar{p} \tau_{u} \tilde{\mathfrak{p}}+\tau_{r} \tilde{\mathfrak{r}}+\tau_{u} \tilde{\mathfrak{e}}$ form a system of two coupled wave equations with common sound speed $c=\tau_{r}+\tau \tau_{u}$, while $\tilde{S}=\beta(\tilde{\mathfrak{e}}-\bar{p} \tilde{\mathfrak{p}}-\tau \tilde{\tau})$ does not evolve in time.

\section{Equilibrium fluctuation}

In this section, let $H(t, x)$ be a bounded and smooth function on $[0, \infty) \times[0,1]$. For any $T>0$, we define two norms $|H|_{T}$ and $\|H\|_{T}$ of $H$ as below:

$$
\begin{gathered}
|H|_{T}=\sup _{[0, T] \times[0,1]}|H(t, x)| \\
\|H\|_{T}^{2}=\sup _{t \in[0, T]}\|H(t)\|^{2}=\sup _{t \in[0, T]} \int_{0}^{1}|H(t, x)|^{2} d x .
\end{gathered}
$$

For $Y_{N}(t, H(t, \cdot))$, the following decomposition holds $\mathbb{P}_{\lambda, N}$ almost surely:

$$
\begin{aligned}
& Y_{N}(t, H(t))-Y_{N}(0, H(0))-\int_{0}^{t} Y_{N}\left(s, \partial_{s} H(s)\right) d s \\
= & I_{N, 1}(t, H)+\gamma I_{N, 2}(t, H)+\sqrt{\gamma} M_{N}(t, H), \quad \forall t>0,
\end{aligned}
$$

where $I_{N, 1}$ and $I_{N, 2}$ are integrals given by

$$
I_{N, 1}(t, H)=N \int_{0}^{t} \mathcal{A}_{N}\left[Y_{N}(s, H(s))\right] d s, \quad I_{N, 2}(t, H)=N \int_{0}^{t} \mathcal{S}_{N}\left[Y_{N}(s, H(s))\right] d s,
$$


and $M_{N}$ is a martingale with quadratic variation given by

$$
\left\langle M_{N}\right\rangle(t, H)=N \int_{0}^{t}\left\{\mathcal{S}_{N}\left[Y_{N}^{2}(s, H(s))\right]-2 Y_{N}(s, H(s)) \mathcal{S}_{N}\left[Y_{N}(s, H(s))\right]\right\} d s .
$$

As the first step to prove Theorem 3.3 , the next lemma guarantees that the last two terms in 4.1) vanish uniformly in macroscopic time for equilibrium dynamics.

Lemma 4.1. There exists a constant $C=C(\lambda, V)$, such that

$$
\mathbb{E}_{\lambda, N}\left[\sup _{t \in[0, T]} \gamma\left|I_{N, 2}(t, H)\right|^{2}+\sup _{t \in[0, T]}\left|M_{N}(t, H)\right|^{2}\right] \leq \frac{C T}{N}\left\|\partial_{x} H\right\|_{T}^{2} .
$$

The proof of Lemma 4.1 is standard and we postpone it to the end of this section. To identify the boundary conditions of $H$, noting that $p_{0}=0$, and

$$
\begin{aligned}
& N \mathcal{A}_{N}\left[Y_{N}(t, H(t))\right] \\
= & \sqrt{N} \sum_{i=1}^{N-1} H\left(t, \frac{i}{N}\right) \cdot\left(J_{\mathcal{A}, i}-J_{\mathcal{A}, i-1}\right)+\sqrt{N} H(t, 1) \cdot\left(\begin{array}{c}
\tau-V\left(r_{N}\right) \\
p_{N}-p_{N-1} \\
p_{N} \tau-p_{N-1} V^{\prime}\left(r_{N}\right)
\end{array}\right) \\
= & \frac{1}{\sqrt{N}} \sum_{i=1}^{N-1} \nabla_{N, i} H(t) \cdot\left(-J_{\mathcal{A}, i}\left(\eta_{t}\right)\right) \\
& -\sqrt{N}\left[H_{1}\left(t, \frac{1}{N}\right)\left(V^{\prime}\left(r_{1}(t)\right)-\tau\right)-\left(H_{2}(t, 1)+\tau H_{3}(t, 1)\right) p_{N}(t)\right],
\end{aligned}
$$

where $J_{\mathcal{A}, i}$ is the centered instantaneous currents of $\mathcal{A}_{N}$ :

$$
J_{\mathcal{A}, i}=\left(V^{\prime}\left(r_{i+1}\right)-\tau, p_{i}, p_{i} V^{\prime}\left(r_{i+1}\right)\right)^{T},
$$

and $\nabla_{N, i}$ is the discrete derivative operator:

$$
\nabla_{N, i} H=N\left[H\left(\frac{i+1}{N}\right)-H\left(\frac{i}{N}\right)\right] .
$$

Thus, we can drop the right boundary if $H(t) \in \mathscr{C}_{*}(\tau)$ for all $t$ :

$$
I_{N, 1}(t, H)=-\frac{1}{\sqrt{N}} \int_{0}^{t} \sum_{i=0}^{N-1} \nabla_{N, i} H(s) \cdot J_{\mathcal{A}, i}\left(\eta_{s}\right) d s .
$$

The next lemma shows that $I_{N, 1}$ can be linearized as $N \rightarrow \infty$.

Lemma 4.2. Assume 2.1], (3.13), and $H(t) \in \mathscr{C}_{*}(\tau)$ for $t \in[0, T]$, then

$$
\mathbb{E}_{\lambda, N}\left[\sup _{t \in[0, T]}\left|I_{N, 1}(t, H)-\int_{0}^{t} Y_{N}\left(s, L^{*} H(s)\right) d s\right|^{2}\right] \leq C\left(\frac{T}{N^{\frac{1}{5}}}+\frac{T^{2}}{N^{\frac{2}{5}}}\right)
$$

holds with some constant $C$. Furthermore,

$$
C \leq C(\lambda, \gamma, V)\|H\|_{T}^{2}, \quad \text { where } \quad\|H\|_{T}^{2}=\left|\partial_{x} H\right|_{T}^{2}+\left|\partial_{x}^{2} H\right|_{T}^{2}+\left\|\partial_{x} H\right\|_{T}^{2} .
$$

Remark 4.3. The bound 4.3 in Lemma 4.2 is proven under the assumption (3.13). Without assuming 3.13 we have only that, for every fixed $T>0$,

$$
\mathbb{E}_{\lambda, N}\left[\sup _{t \in[0, T]}\left|I_{N, 1}(t, H)-\int_{0}^{t} Y_{N}\left(s, L^{*} H(s)\right) d s\right|^{2}\right] \leq o_{N}(1)\|H\|_{T}^{2} .
$$

This is clear from Remark 5.3 below. The bound (4.4) is enough for proving Theorem 3.1 while 4.3 is necessary in order to prove (3.14). 
Lemma 4.2 follows from the Boltzmann-Gibbs principle, proven in Section 5. Here we first give the proof of Theorem 3.3 .

Proof of Theorem 3.3. Let $H(t, x)$ be the solution of (3.10). From (4.1) and Lemma 4.1.

$$
\begin{aligned}
\mathbb{P}_{\lambda, N}\{\exists t \in[0, T], & \mid Y_{N}\left(N^{\alpha} t, H\left(N^{\alpha} t\right)\right)-Y_{N}(0, H(0)) \\
& \left.-\int_{0}^{N^{\alpha} t} Y_{N}\left(s, \partial_{s} H(s)\right) d s-I_{N, 1}\left(N^{\alpha} t, H\right) \mid>\epsilon\right\} \rightarrow 0
\end{aligned}
$$

for any $\epsilon>0$. Lemma 4.2 and $(3.12)$ then yield that for any $\alpha<1 / 5$,

$$
\mathbb{E}_{\lambda, N}\left[\sup _{t \in[0, T]}\left|I_{N, 1}\left(N^{\alpha} t, H\right)-\int_{0}^{N^{\alpha} t} Y_{N}\left(s, L^{*} H(s)\right) d s\right|^{2}\right] \rightarrow 0 .
$$

Theorem 3.3 then follows from 3.10 .

For Theorem 3.1, since tightness is shown in Section 7, we only need to take $\alpha=0$ in the proof above, and apply Remark 4.3 instead of Lemma 4.2 in the last step.

We now proceed to the proof of Lemma 4.1. Denote by $\langle\cdot, \cdot\rangle_{\lambda, N}$ the scalar product of two functions $f, g \in L^{2}\left(\pi_{\lambda, N}\right)$. We make use of a well-known estimate on the space-time variance of a stationary Markov process. For $f(s, \cdot) \in L^{2}\left(\pi_{\lambda, N}\right)$,

$$
\mathbb{E}_{\lambda, N}\left[\sup _{t \in[0, T]}\left|\int_{0}^{t} f\left(s, \eta_{s}\right) d s\right|^{2}\right] \leq 14 \int_{0}^{T}\|f(t)\|_{-1, N}^{2} d t,
$$

where $\|f\|_{-1, N}$ is defined for all $f$ on $\Omega_{N}$ by

$$
\|f\|_{-1, N}^{2}=\sup _{h}\left\{2\langle f, h\rangle_{\lambda, N}-\gamma N\left\langle h,-\mathcal{S}_{N} h\right\rangle_{\lambda, N}\right\}
$$

with the superior taken over all bounded smooth functions $h$ on $\Omega_{N}$. A proof of 4.5 can be found in [14, Sec. 2.5].

Proof of Lemma 4.1. To begin with, note that

$$
\begin{aligned}
N \mathcal{S}_{N}\left[Y_{N}(t, H(t))\right] & =\frac{\sqrt{N}}{2} \sum_{i=1}^{N-1}\left[H\left(t, \frac{i}{N}\right) \cdot \mathcal{Y}_{i, i+1}^{2}\left[w_{i}\right]+H\left(t, \frac{i+1}{N}\right) \cdot \mathcal{Y}_{i, i+1}^{2}\left[w_{i+1}\right]\right] \\
& =-\frac{1}{\sqrt{N}} \sum_{i=1}^{N-1} \nabla_{N, i} H(t) \cdot J_{\mathcal{S}, i}\left(\eta_{t}\right),
\end{aligned}
$$

where $J_{\mathcal{S}, i}$ is the instantaneous current corresponding to $\mathcal{S}_{N}$ :

$$
J_{\mathcal{S}, i}=\frac{1}{2} \mathcal{Y}_{i, i+1}^{2}\left[w_{i}\right]=-\frac{1}{2} \mathcal{Y}_{i, i+1}^{2}\left[w_{i+1}\right]
$$

By applying 4.5 on $I_{N, 2}(t, H)$, one obtains that

$$
\begin{aligned}
& \mathbb{E}_{\lambda, N}\left[\sup _{t \in[0, T]}\left|I_{N, 2}(t, H)\right|^{2}\right] \\
\leq & \frac{14}{N} \int_{0}^{T} \sup _{h}\left\{2 \sum_{i=1}^{N-1}\left\langle\nabla_{N, i} H(t) \cdot J_{\mathcal{S}, i}, h\right\rangle_{\lambda, N}-\gamma N\left\langle h,-\mathcal{S}_{N} h\right\rangle_{\lambda, N}\right\} d t .
\end{aligned}
$$


By Cauchy-Schwarz inequality, with $m_{i}=\mathcal{Y}_{i, i+1}\left[w_{i}\right]$ for $i=1$ to $N-1$,

$$
\begin{aligned}
\left|\sum_{i=1}^{N-1}\left\langle\nabla_{N, i} H(t) \cdot J_{\mathcal{S}, i}, h\right\rangle_{\lambda, N}\right|^{2} & =\frac{1}{4}\left|\sum_{i=1}^{N-1}\left\langle\nabla_{N, i} H(t) \cdot m_{i}, \mathcal{Y}_{i, i+1} h\right\rangle_{\lambda, N}\right|^{2} \\
& \leq \frac{1}{4} \sum_{i=1}^{N-1}\left|\nabla_{N, i} H(t)\right|^{2} E_{\lambda, N}\left[\left|m_{i}\right|^{2}\right] \sum_{i=1}^{N-1} E_{\lambda, N}\left[\left|\mathcal{Y}_{i, i+1} h\right|^{2}\right] \\
& \leq C_{1} N\left\|\partial_{x} H(t)\right\|^{2}\left\langle h,-\mathcal{S}_{N} h\right\rangle_{\lambda, N} .
\end{aligned}
$$

Substituting this and optimizing $h$, we obtain that

$$
\mathbb{E}_{\lambda, N}\left[\sup _{t \in[0, T]}\left|I_{N, 2}(t, H)\right|^{2}\right] \leq \frac{14 C_{1}}{\gamma N} \int_{0}^{T}\left\|\partial_{x} H\right\|^{2} d t \leq \frac{C_{2} T}{\gamma N}\left\|\partial_{x} H\right\|_{T}^{2} .
$$

On the other hand, recall that $m_{i}=\mathcal{Y}_{i, i+1}\left[w_{i}\right]$ and

$$
\mathcal{S}_{N}\left[Y_{N}^{2}(s, H(s))\right]-2 Y_{N}(s, H(s)) \mathcal{S}_{N}\left[Y_{N}(s, H(s))\right]=\frac{1}{N^{3}} \sum_{i=1}^{N-1}\left[\nabla_{N, i} H(s) \cdot m_{i}\left(\eta_{s}\right)\right]^{2} .
$$

Therefore, by Doob's maximal inequality,

$$
\begin{aligned}
\mathbb{E}_{\lambda, N}\left[\sup _{t \in[0, T]}\left|M_{N}(t, H)\right|^{2}\right] & \leq 4 \mathbb{E}_{\lambda, N}\left[\left\langle M_{N}\right\rangle(T, H)\right] \\
& \leq \frac{4}{N^{2}} \int_{0}^{T} \sum_{i=1}^{N-1} E_{\lambda, N}\left[\left(\nabla_{N, i} H(t) \cdot m_{i}\right)^{2}\right] d t \\
& \leq \frac{C_{3}}{N} \int_{0}^{T}\left\|\partial_{x} H\right\|^{2} d t \leq \frac{C_{4} T}{N}\left\|\partial_{x} H\right\|_{T}^{2} .
\end{aligned}
$$

Since the constants depend only on $\lambda$ and $V$, Lemma 4.1 follows.

\section{Boltzmann-Gibbs principle}

This section devotes to the proof of Lemma 4.2 . In this section, we denote by $\left\{\iota_{i} ; 0 \leq i \leq N\right\}$ the shift operator semigroup on $\Omega_{N}$, which is given by

$$
\left(\iota_{i} \eta\right)_{j}= \begin{cases}\left(p_{i+j}, r_{i+j}\right), & 1 \leq j \leq N-i \\ (0,0), & N-i<j \leq N\end{cases}
$$

for all $\eta \in \Omega_{N}$ and $0 \leq i \leq N$. For function $F$ on $\Omega_{N}$, define $\iota_{i} F=F \circ \iota_{i}$. If $F$ is supported by $\left\{\eta_{j}, 1 \leq j \leq m\right\}$ for some $m \leq N$, then

$$
E_{\lambda, N}\left[\iota_{i} F\right]=E_{\lambda, N}[F], \quad \forall 0 \leq i \leq N-m .
$$

First notice that $\nabla_{N, i} H$ in 4.2 can be replaced by $\partial_{x} H$. The difference is

$$
\begin{aligned}
& \mathbb{E}_{\lambda, N}\left[\sup _{t \in[0, T]}\left|I_{N, 1}(t, H)-\frac{1}{\sqrt{N}} \int_{0}^{t} \sum_{i=1}^{N-1} \partial_{x} H\left(s, \frac{i}{N}\right) \cdot J_{\mathcal{A}, i}\left(\eta_{s}\right) d s\right|^{2}\right] \\
\leq & \frac{T}{N} \int_{0}^{T} E_{\lambda, N}\left[\left(\sum_{i=1}^{N-1}\left[\nabla_{N, i} H(t)-\partial_{x} H\left(t, \frac{i}{N}\right)\right] \cdot J_{\mathcal{A}, i}\right)^{2}\right] d t .
\end{aligned}
$$


Since $E_{\lambda, N}\left[J_{\mathcal{A}, i} \otimes J_{\mathcal{A}, j}\right]=\mathbf{0}$ if $|i-j|>1$, where $\otimes$ is the tensor product of vectors, the last line in the inequality above has an upper bound

$$
\frac{T}{N} E_{\lambda, N}\left[\left|J_{\mathcal{A}, i}\right|^{2}\right] \int_{0}^{T} \sum_{i=1}^{N-1}\left|\nabla_{N, i} H(t)-H^{\prime}\left(t, \frac{i}{N}\right)\right|^{2} d t \leq \frac{C T^{2}\left|\partial_{x}^{2} H\right|_{T}^{2}}{N^{2}}
$$

Clearly the order is better than what is needed for Lemma 4.2 .

Now we want to replace the local random field $J_{\mathcal{A}, i}$ with its linear approximation. The corresponding error can be expressed by

$$
\iota_{i} \Phi=J_{\mathcal{A}, i}-B(\lambda)\left(w_{i}-\bar{w}(\lambda)\right)=\left(\begin{array}{c}
V^{\prime}\left(r_{i+1}\right)-\tau_{r} r_{i}-\tau_{e} e_{i} \\
0 \\
p_{i} V^{\prime}\left(r_{i+1}\right)-p_{i} \tau
\end{array}\right) .
$$

Lemma 4.2 follows from the following Boltzmann-Gibbs principle.

Proposition 5.1. Assume (2.1) and 3.13 , then

$$
\mathbb{E}_{\lambda, N}\left[\sup _{t \in[0, T]}\left|\frac{1}{\sqrt{N}} \int_{0}^{t} \sum_{i=1}^{N-1} \partial_{x} H\left(s, \frac{i}{N}\right) \cdot \iota_{i} \Phi\left(\eta_{s}\right) d s\right|^{2}\right] \leq C\left(\frac{T}{N^{\frac{1}{5}}}+\frac{T^{2}}{N^{\frac{2}{5}}}\right)
$$

for bounded smooth $H=H(t, x)$ on $[0, T] \times[0,1]$, where $C=C(\lambda, \gamma, V)\|H\|_{T}^{2}$.

Boltzmann-Gibbs principle, firstly established for zero range jump process (see [7]), aims at determining the space-time fluctuation of a local function by its linear approximation on the conserved fields. To show this proposition, we need a spectral gap bound of $\mathcal{S}_{N}$, which is the main difficulty here. This is established later in Section 6 .

Remark 5.2. Notice that the upper bound in Proposition 5.1 is not optimal. Indeed, with the proof below, one can actually obtain an upper bound of

$$
C\left(\frac{T}{N^{1-2 b}}+\frac{T^{2}}{N^{b}}\right), \quad \forall b<\frac{1}{2}
$$

However, this does not improve the time scale in Theorem 3.3

Proof. The first step is to take some $1 \leq K \ll N$, and define

$$
\Phi_{K}=\frac{1}{K} \sum_{i=1}^{K} \iota_{i} \Phi
$$

We want to replace $\iota_{i} \Phi$ by $\iota_{i-1} \Phi_{K}$. The error is

$$
\sum_{i=1}^{N-1} a_{i}(t) \cdot \iota_{i} \Phi-\sum_{i=1}^{N-K} a_{i}(t) \cdot \iota_{i-1} \Phi_{K}=F_{1}(t)+F_{2}(t)
$$

where we write $a_{i}(t)=\partial_{x} H(t, i / N)$ for short, and $F_{1}, F_{2}$ are given by

$$
\begin{aligned}
& F_{1}(t)=\frac{1}{K}\left(\sum_{i=1}^{K-1}+\sum_{i=N-K+1}^{N-1}\right)(K-i) a_{i}(t) \cdot \iota_{i} \Phi \\
& F_{2}(t)=\frac{1}{K}\left(\sum_{i=1}^{K} \sum_{j=1}^{i}+\sum_{i=K+1}^{N-K-1} \sum_{j=i+1-K}^{i}+\sum_{i=N-K}^{N-1} \sum_{j=i+1-K}^{N-K}\right)\left(a_{i}(t)-a_{j}(t)\right) \cdot \iota_{i} \Phi .
\end{aligned}
$$


Since $E_{\lambda, N}\left[\iota_{i} \Phi \otimes \iota_{j} \Phi\right]=\mathbf{0}$ for every pair of $(i, j)$ such that $|i-j| \geq 2$,

$$
E_{\lambda, N}\left[F_{1}^{2}(t)+F_{2}^{2}(t)\right] \leq\left(C_{1}+\frac{C_{2}}{N}\right) K\left(\left|\partial_{x} H\right|_{T}^{2}+\left|\partial_{x}^{2} H\right|_{T}^{2}\right),
$$

with constants $C_{1}$ and $C_{2}$ depending on $\lambda$ and $V$. Hence,

$$
\mathbb{E}_{\lambda, N}\left[\sup _{t \in[0, T]}\left|\frac{1}{\sqrt{N}} \int_{0}^{t} F_{1}\left(s, \eta_{s}\right)+F_{2}\left(s, \eta_{s}\right) d s\right|^{2}\right] \leq \frac{C_{3} T^{2} K}{N}\left(\left|\partial_{x} H\right|_{T}^{2}+\left|\partial_{x}^{2} H\right|_{T}^{2}\right) .
$$

The second step is to replace $\Phi_{K}$ by its microcanonical center. To do so, observe that $\Phi_{K}$ is supported by $\left\{\eta_{j} ; 1 \leq j \leq K+1\right\}$, and define

$$
\left\langle\Phi_{K}\right\rangle=E_{\lambda, N}\left[\Phi \mid \frac{w_{1}+w_{2}+\ldots+w_{K+1}}{K+1}\right],
$$

where $w_{i}=\left(p_{i}, r_{i}, e_{i}\right)$ is the vector if conserved quantities. Due to the equivalence of ensembles (see Section 8), the second moment of $\left\langle\Phi_{K}\right\rangle$ with respect to $\pi_{\lambda, N}$ is of order $K^{-2}$. On the other hand, the second moment of $\Phi_{K}$ is $O\left(K^{-1}\right)$ :

$$
E_{\lambda, N}\left[\left|\Phi_{K}\right|^{2}\right] \leq \frac{1}{K}\left(E_{\lambda, N}\left[\left|\iota_{1} \Phi\right|^{2}\right]+2 E_{\lambda, N}\left[\iota_{1} \Phi \cdot \iota_{2} \Phi\right]\right),
$$

Define $\varphi_{K}=\Phi_{K}-\left\langle\Phi_{K}\right\rangle$. Since $\varphi_{K}$ and $\left\langle\Phi_{K}\right\rangle$ are orthogonal,

$$
E_{\lambda, N}\left[\left|\varphi_{K}\right|^{2}\right]=E_{\lambda, N}\left[\left|\Phi_{K}\right|^{2}\right]-E_{\lambda, N}\left[\left|\left\langle\Phi_{K}\right\rangle\right|^{2}\right] \leq \frac{C_{4}}{K} .
$$

By applying the estimate (4.5), we obtain that

$$
\begin{aligned}
& \mathbb{E}_{\lambda, N}\left[\sup _{t \in[0, T]}\left|\frac{1}{\sqrt{N}} \int_{0}^{t} \sum_{i=1}^{N-K} a_{i}(s) \cdot \iota_{i-1} \varphi_{K}\left(\eta_{s}\right) d s\right|^{2}\right] \\
\leq & \frac{14}{N} \int_{0}^{T} \sup _{h}\left\{2 \sum_{i=1}^{N-K}\left\langle a_{i}(t) \cdot \iota_{i-1} \varphi_{K}, h\right\rangle_{\lambda, N}-\gamma N\left\langle h,-\mathcal{S}_{N} h\right\rangle_{\lambda, N}\right\} d t,
\end{aligned}
$$

where the superior is taken over all bounded smooth functions on $\Omega_{N}$. As $\varphi_{K}$ is supported by $\left\{\eta_{i} ; 1 \leq i \leq K+1\right\}$, by the spectral gap in Proposition 6.1.

$$
-\mathcal{S}_{K+1} G_{a, K}=a \cdot \varphi_{K}, \quad a \in \mathbb{R}^{3}
$$

can be solved by some function $G_{a, K}$ satisfying that

$$
\left\langle G_{a, K},-\mathcal{S}_{K+1} G_{a, K}\right\rangle_{\lambda, N} \leq C(K+1)^{2} E_{\lambda, N}\left[\left(a \cdot \varphi_{K}\right)^{2}\right] \leq C_{5} K|a|^{2},
$$

where the last step follows from 5.2 . For $1 \leq i \leq N-K$ and $a \in \mathbb{R}^{3}$,

$$
\left\langle a \cdot \iota_{i-1} \varphi_{K}, h\right\rangle_{\lambda, N}=\frac{1}{2} \sum_{j=1}^{K}\left\langle\mathcal{Y}_{i+j-1, i+j}\left[\iota_{i-1} G_{a, K}\right], \mathcal{Y}_{i+j-1, i+j} h\right\rangle_{\lambda, N} .
$$


Hence, by Cauchy-Schwarz inequality,

$$
\begin{aligned}
& \left|\sum_{i=1}^{N-K}\left\langle a_{i}(t) \cdot \iota_{i-1} \varphi_{K}, h\right\rangle_{\lambda, N}\right|^{2} \\
\leq & \left(\frac{1}{2} \sum_{i=1}^{N-K} \sum_{j=1}^{K} E_{\lambda, N}\left[\left(\mathcal{Y}_{i+j-1, i+j} h\right)^{2}\right]\right)\left(\frac{1}{2} \sum_{i=1}^{N-K} \sum_{j=1}^{K} E_{\lambda, N}\left[\left(\mathcal{Y}_{j, j+1} G_{a_{i}(t), K}\right)^{2}\right]\right) \\
\leq & K\left\langle h,-\mathcal{S}_{N} h\right\rangle_{\lambda, N} \sum_{i=1}^{N-K}\left\langle G_{a_{i}(t), K},-\mathcal{S}_{K+1} G_{a_{i}(t), K}\right\rangle_{\lambda, N} \\
\leq & C_{5} K^{2}\left\langle h,-\mathcal{S}_{N} h\right\rangle_{\lambda, N} \sum_{i=1}^{N-K}\left|a_{i}(t)\right|^{2} \leq C_{6} K^{2} N\left\|\partial_{x} H(t)\right\|^{2}\left\langle h,-\mathcal{S}_{N} h\right\rangle_{\lambda, N} .
\end{aligned}
$$

Substituting this into 5.3 and optimizing in $h$,

$$
\mathbb{E}_{\lambda, N}\left[\sup _{t \in[0, T]}\left|\frac{1}{\sqrt{N}} \int_{0}^{t} \sum_{i=1}^{N-K} a_{i}(s) \cdot \iota_{i-1} \varphi_{K}\left(\eta_{s}\right) d s\right|^{2}\right] \leq \frac{C_{7} T K^{2}}{\gamma N}\left\|\partial_{x} H\right\|_{T}^{2} .
$$

Finally, $\left\langle\Phi_{K}\right\rangle$ is supported by $\left\{\eta_{i} ; 1 \leq i \leq K+1\right\}$, so that $E_{\lambda, N}\left[\iota_{i}\left\langle\Phi_{K}\right\rangle \otimes \iota_{j}\left\langle\Phi_{K}\right\rangle\right]=0$ for $|i-j| \geq K+2$, and therefore,

$$
\begin{aligned}
& \mathbb{E}_{\lambda, N}\left[\sup _{t \in[0, T]}\left|\frac{1}{\sqrt{N}} \int_{0}^{t} \sum_{i=1}^{N-K} a_{i}(s) \cdot \iota_{i-1}\left\langle\Phi_{K}\right\rangle\left(\eta_{s}\right) d s\right|^{2}\right] \\
\leq & \frac{T}{N} \int_{0}^{T} \sum_{|i-j| \leq K+1} E_{\lambda, N}\left[\left(a_{i}(t) \cdot \iota_{i-1}\left\langle\Phi_{K}\right\rangle\right)\left(a_{j}(t) \cdot \iota_{j-1}\left\langle\Phi_{K}\right\rangle\right)\right] d t \\
\leq & \frac{T^{2}\left\|\partial_{x} H\right\|_{T}^{2}}{N} \sum_{i=1}^{N-K} \sum_{j=-K-1}^{K+1} E_{\lambda, N}\left[\left|\left\langle\Phi_{K}\right\rangle \| \iota_{j}\left\langle\Phi_{K}\right\rangle\right|\right] \leq \frac{C_{8} T^{2}}{K}\left\|\partial_{x} H\right\|_{T}^{2},
\end{aligned}
$$

where the last line is due to that $E_{\lambda, N}\left[\left\langle\Phi_{K}\right\rangle^{2}\right]=O\left(K^{-2}\right)$.

In conclusion, by summing up (5.1), (5.4), (5.5), and taking $K=N^{2 / 5}$, we get the estimate in Proposition 5.1. with the constant satisfying that

$$
C \leq C(\lambda, \gamma, V)\left(\left\|\partial_{x} H\right\|_{T}^{2}+\left|\partial_{x} H\right|_{T}^{2}+\left|\partial_{x}^{2} H\right|_{T}^{2}\right)
$$

This completes the proof of the proposition.

Remark 5.3. If only (2.1) is assumed, we can apply Remark 6.6 instead of Proposition 6.1 in the proof of (5.4). By doing this, we can prove Proposition 5.1 for any fixed $T>0$, with a weaker upper bound $o_{N}(1)\|H\|_{T}^{2}$.

\section{Spectral gap}

In this section, we state and prove the spectral gap estimate for the dynamics. The main result, Proposition 6.1, plays a central role in the proof of Proposition 5.1.

Since we want to consider dynamics without boundary conditions in this section, the notations would be slightly different. Recall 2.1 and denote

$$
\delta_{-}=\inf _{r \in \mathbb{R}} V^{\prime \prime}(r), \quad \delta_{+}=\sup _{r \in \mathbb{R}} V^{\prime \prime}(r) .
$$


For $\beta>0,(\bar{p}, \tau) \in \mathbb{R}^{2}$, let $\pi_{\beta, \bar{p}, \tau}^{K}$ be the product measure on $\Omega_{K}$ given by

$$
\pi_{\beta, \bar{p}, \tau}^{K}(d \mathbf{p} d \mathbf{r})=\prod_{i=1}^{K} \frac{1}{Z_{\beta, \tau}} \exp \left\{-\frac{\beta\left(p_{i}-\bar{p}\right)^{2}}{2}-\beta V\left(r_{i}\right)+\beta \tau r_{i}\right\} d p_{i} d r_{i},
$$

where $Z_{\beta, \tau}$ is the normalization constant. Note that the additional coefficient $\bar{p}$ refers to a nonzero average speed. For $K \geq 2$ and $w=(p, r, e)$ such that $e>p^{2} / 2+V(r)$, the microcanonical manifold $\Omega_{w, K}$ is defined as

$$
\Omega_{w, K}=\left\{\begin{array}{l|l}
\left(p_{k}, r_{k}\right), 1 \leq k \leq K & \frac{1}{K} \sum_{k=1}^{K} w_{k}=w
\end{array}\right\} .
$$

In view of 2.1), $\Omega_{w, K}$ is a compact and connected manifold. The microcanonical expectation on $\Omega_{w, K}$ is defined as the conditional expectation

$$
E_{w, K}=E_{\pi_{\beta, \bar{p}, \tau}^{K}}\left[\cdot \mid \Omega_{w, K}\right]
$$

Notice that the definition of $E_{w, K}$ is independent of the choice of $\beta, \bar{p}$ or $\tau$. For two functions $f_{1}, f_{2}$ such that $E_{w, K}\left[f_{i}^{2}\right]<\infty$, we write $\left\langle f_{1}, f_{2}\right\rangle_{w, K}=E_{w, K}\left[f_{1} f_{2}\right]$. For each pair $(i, j)$ such that $1 \leq i<j \leq K$, let $\mathscr{F}_{i, j}$ be the $\sigma$-algebra over $\Omega_{w, K}$ given by

$$
\mathscr{F}_{i, j}=\sigma\left(\left\{\left(p_{k}, r_{k}\right) ; 1 \leq k \leq K, k \neq i, j\right\}\right) .
$$

Proposition 6.1. Suppose that the potential $V$ satisfies (2.1). There exists a universal constant $\delta>0$, such that if $V$ fulfills furthermore 3.13 , then

$$
E_{w, K}\left[\left(f-E_{w, K}[f]\right)^{2}\right] \leq C_{K} \sum_{k=1}^{K-1} E_{w, K}\left[\left(\mathcal{Y}_{k, k+1} f\right)^{2}\right]
$$

for all $(w, K)$ and bounded smooth function $f$, and $C_{K} \leq C K^{2}$.

The proof of Proposition 6.1 is divided into Lemma 6.2, 6.3 and 6.4 below.

Lemma 6.2. Assume 2.1], then there exists constant $C$, such that

$$
E_{w, 2}\left[\left(f-E_{w, 2}[f]\right)^{2}\right] \leq C E_{w, 2}\left[\left(\mathcal{Y}_{1,2} f\right)^{2}\right]
$$

for all $w$ and bounded smooth function $f$ on $\left(p_{1}, r_{1}, p_{2}, r_{2}\right)$.

Lemma 6.3. Assume 2.1, then there exists constant $C$, such that

$$
\sum_{1 \leq i<j \leq K} E_{w, K}\left[\left(f-E_{w, K}\left[f \mid \mathscr{F}_{i, j}\right]\right)^{2}\right] \leq C K^{3} \sum_{k=1}^{K-1} E_{w, K}\left[\left(f-E_{w, K}\left[f \mid \mathscr{F}_{k, k+1}\right]\right)^{2}\right]
$$

for all $K \geq 3, w$ and bounded smooth function $f$.

Lemma 6.4. Assume 2.1) and (3.13), then

$$
E_{w, K}\left[\left(f-E_{w, K}[f]\right)^{2}\right] \leq C_{K}^{\prime} \sum_{1 \leq i<j \leq K} E_{w, K}\left[\left(f-E_{w, K}\left[f \mid \mathscr{F}_{i, j}\right]\right)^{2}\right]
$$

for all $K \geq 3, w$ and bounded smooth function $f$, and $C_{K}^{\prime} \leq C^{\prime} K^{-1}$. 
Indeed, for each $k=1, \ldots, K-1$, by applying Lemma 6.2 to the space $\left(p_{k}, r_{k}, p_{k+1}, r_{k+1}\right)$ and the operator $\mathcal{Y}_{k, k+1}$, one obtains that that

$$
E_{w, K}\left[\left(f-E_{w, K}\left[f \mid \mathscr{F}_{k, k+1}\right]\right)^{2} \mid \mathscr{F}_{k, k+1}\right] \leq C E_{w, K}\left[\left(\mathcal{Y}_{k, k+1} f\right)^{2} \mid \mathscr{F}_{k, k+1}\right] .
$$

Then, Proposition 6.1 turns to be the direct consequence of this, Lemma 6.3 and Lemma 6.4 We now prove these lemmas in turn.

Proof of Lemma 6.2. For $\left(p_{1}, r_{1}, p_{2}, r_{2}\right) \in \mathbb{R}^{4}$, define

$$
p=p\left(p_{1}, p_{2}\right)=\frac{p_{1}+p_{2}}{2}, \quad r=r\left(r_{1}, r_{2}\right)=\frac{r_{1}+r_{2}}{2},
$$

and the internal energy $E=E\left(p_{1}, r_{1}, p_{2}, r_{2}\right) \geq 0$ given by

$$
E=\frac{e_{1}+e_{2}}{2}-\frac{p^{2}}{2}-V(r)=\frac{\left(p_{1}-p_{2}\right)^{2}}{8}+\frac{V\left(r_{1}\right)+V\left(r_{2}\right)}{2}-V\left(\frac{r_{1}+r_{2}}{2}\right) .
$$

Furthermore, let $\theta \in[0,2 \pi)$ satisfy that $\sqrt{E} \cos \theta=\sqrt{2}\left(p_{1}-p_{2}\right) / 4$ and

$$
\sqrt{E} \sin \theta=\operatorname{sgn}\left(r_{1}-r_{2}\right) \sqrt{\frac{V\left(r_{1}\right)+V\left(r_{2}\right)}{2}-V\left(\frac{r_{1}+r_{2}}{2}\right)} .
$$

The Jacobian determinant of the bijection $\left(p_{1}, r_{1}, p_{2}, r_{2}\right) \rightarrow(p, r, E, \theta)$ is

$$
\mathfrak{J}(p, r, E, \theta)=\sqrt{2} \cdot \frac{\sqrt{V\left(r_{1}\right)+V\left(r_{2}\right)-2 V(r)}}{\left|V^{\prime}\left(r_{1}\right)-V^{\prime}\left(r_{2}\right)\right|} .
$$

Recall that $0<\delta_{-} \leq V^{\prime \prime}(r) \leq \delta_{+}<\infty$, we have

$$
0<\frac{\sqrt{\delta_{-}}}{\sqrt{2} \delta_{+}} \leq \mathfrak{J}(p, r, E, \theta) \leq \frac{\sqrt{\delta_{+}}}{\sqrt{2} \delta_{-}} .
$$

For a bounded smooth function $f=f\left(p_{1}, r_{1}, p_{2}, r_{2}\right)$, define $f_{*}(p, r, E, \theta)=f\left(p_{1}, r_{1}, p_{2}, r_{2}\right)$, and let $\left\langle f_{*}\right\rangle=\int_{0}^{2 \pi} f_{*}(p, r, E, \theta) d \theta$. By simple calculations,

$$
E_{w, 2}\left[\left(f-\left\langle f_{*}\right\rangle\right)^{2}\right]=\frac{\int_{0}^{2 \pi}\left[f_{*}(p, r, E, \theta)-\left\langle f_{*}\right\rangle\right]^{2} \mathfrak{J}(p, r, E, \theta) d \theta}{\int_{0}^{2 \pi} \mathfrak{J}(p, r, E, \theta) d \theta} .
$$

On the other hand, since $\mathcal{Y}_{1,2} f=\mathfrak{J}^{-1} \partial_{\theta} f_{*}$, we have

$$
E_{w, 2}\left[\left(\mathcal{Y}_{1,2} f\right)^{2}\right]=\frac{\int_{0}^{2 \pi}\left[\partial_{\theta} f_{*}(p, r, E, \theta)\right]^{2} \mathfrak{J}^{-1}(p, r, E, \theta) d \theta}{\int_{0}^{2 \pi} \mathfrak{J}(p, r, E, \theta) d \theta}
$$

By virtue of the Poincaré inequality on one-dimensional torus:

$$
\int_{0}^{2 \pi}\left(f_{*}-\left\langle f_{*}\right\rangle\right)^{2} d \theta \leq C \int_{0}^{2 \pi}\left(\partial_{\theta} f_{*}\right)^{2} d \theta
$$

and the uniform bound of $\mathfrak{J}$ in 6.3 , we obtain that

$$
E_{w, 2}\left[\left(f-E_{w, 2}[f]\right)^{2}\right] \leq E_{w, 2}\left[\left(f-\left\langle f_{*}\right\rangle\right)^{2}\right] \leq \frac{C \delta_{+}}{2 \delta_{-}^{2}} E_{w, 2}\left[\left(X_{1,2} f\right)^{2}\right]
$$

holds with some universal constant $C<\infty$. 
Proof of Lemma 6.3. This lemma is proved along the idea in [17, Lemma 12.4]. Below are some notations only used in this proof. All of the subscripts $i, j, k$ are taken form $\{1, \ldots, K\}$. We write $x_{k}=\left(p_{k}, r_{k}\right)$ and $\mathbf{x}=\left(x_{1}, \ldots, x_{K}\right)$. Recall the bijection defined in the proof of the Lemma 6.2 For simplicity we write

$$
\left(p_{i, j}, r_{i, j}, E(i, j), \theta_{i, j}\right)=(p, r, E, \theta)\left(x_{i}, x_{j}\right), \quad \forall i<j .
$$

For $\theta \in[0,2 \pi)$, denote the Jacobian determinant by

$$
\mathfrak{J}_{\mathbf{x}, i, j}(\theta)=\mathfrak{J}\left(p_{i, j}, r_{i, j}, E(i, j), \theta\right) .
$$

For $i<j, \theta \in[0,2 \pi]$ and $\mathbf{x}=\left(x_{1}, \ldots, x_{K}\right)$, define a vector $\rho_{i, j}^{\theta} \mathbf{x}$ by

$$
\left(\rho_{i, j}^{\theta} \mathbf{x}\right)_{k}= \begin{cases}g_{1}\left(p_{i, j}, r_{i, j}, E(i, j), \theta\right), & k=i \\ g_{2}\left(p_{i, j}, r_{i, j}, E(i, j), \theta\right), & k=j \\ x_{k}, & k \neq i, j,\end{cases}
$$

where $\left(g_{1}, g_{2}\right)$ denotes the inverse map of $\left(x_{1}, x_{2}\right) \rightarrow(p, r, E, \theta)$. Observe that $\rho_{i, j}^{\theta} \mathbf{x}=\mathbf{x}$ when $\theta=\theta_{i, j}$, and for every smooth function $f$,

$$
E_{w, K}\left[f \mid \mathscr{F}_{i, j}\right]=\frac{1}{J_{x_{i}+x_{j}}} \int_{0}^{2 \pi} f\left(\rho_{i, j}^{\theta} \mathbf{x}\right) \mathfrak{J}_{\mathbf{x}, i, j}(\theta) d \theta,
$$

where $J_{x_{i}+x_{j}}=\int_{0}^{2 \pi} \mathfrak{J}_{\mathbf{x}, i, j}(\theta) d \theta$. On the other hand, let $\tau_{i, j} \mathbf{x}$ be the vector given by

$$
\left(\tau_{i, j} \mathbf{x}\right)_{i}=x_{j}, \quad\left(\tau_{i, j} \mathbf{x}\right)_{j}=x_{i}, \quad\left(\tau_{i, j} \mathbf{x}\right)_{k}=x_{k}, \forall k \neq i, j .
$$

Moreover for $1 \leq i<j \leq K$, we inductively define that

$$
\sigma_{i, i}=\tilde{\sigma}_{i, i}=i d, \quad \sigma_{i, j}=\tau_{j-1, j} \circ \sigma_{i, j-1}, \quad \tilde{\sigma}_{i, j}=\tilde{\sigma}_{i, j-1} \circ \tau_{j-1, j} .
$$

Observe that for any $i<j$ and $\theta \in[0,2 \pi), \rho_{i, j}^{\theta} \equiv \tilde{\sigma}_{i, j-1} \circ \rho_{j-1, j}^{\theta} \circ \sigma_{i, j-1}$.

For a smooth function $f$, by Cauchy-Schwarz inequality,

$$
\left(f-E_{w, K}\left[f \mid \mathscr{F}_{i, j}\right]\right)^{2} \leq \frac{1}{J_{x_{i}+x_{j}}} \int_{0}^{2 \pi}\left[f\left(\rho_{i, j}^{\theta} \mathbf{x}\right)-f(\mathbf{x})\right]^{2} \mathfrak{J}_{\mathbf{x}, i, j}(\theta) d \theta .
$$

The right-hand side is bounded from above by $3\left(f_{1}+f_{2}+f_{3}\right)$, where

$$
\begin{aligned}
& f_{1}=\frac{1}{J_{x_{i}+x_{j}}} \int_{0}^{2 \pi}\left[f\left(\sigma_{i, j-1} \mathbf{x}\right)-f(\mathbf{x})\right]^{2} \mathfrak{J}_{\mathbf{x}, i, j}(\theta) d \theta, \\
& f_{2}=\frac{1}{J_{x_{i}+x_{j}}} \int_{0}^{2 \pi}\left[f\left(\rho_{j-1, j}^{\theta} \circ \sigma_{i, j-1} \mathbf{x}\right)-f\left(\sigma_{i, j-1} \mathbf{x}\right)\right]^{2} \mathfrak{J}_{\mathbf{x}, i, j}(\theta) d \theta, \\
& f_{3}=\frac{1}{J_{x_{i}+x_{j}}} \int_{0}^{2 \pi}\left[f\left(\tilde{\sigma}_{i, j-1} \circ \rho_{j-1, j}^{\theta} \circ \sigma_{i, j-1} \mathbf{x}\right)-f\left(\rho_{j-1, j}^{\theta} \circ \sigma_{i, j-1} \mathbf{x}\right)\right]^{2} \mathfrak{J}_{\mathbf{x}, i, j}(\theta) d \theta .
\end{aligned}
$$

For $f_{1}$, noticing that $f_{1}=\left(f\left(\sigma_{i, j-1} \mathbf{x}\right)-f(\mathbf{x})\right)^{2}$, hence

$$
\begin{aligned}
E_{w, K}\left[f_{1}\right] & \leq K \sum_{k=i}^{j-2} E_{w, K}\left[\left(f \circ \sigma_{i, k+1}-f \circ \sigma_{i, k}\right)^{2}\right] \\
& =K \sum_{k=i}^{j-2} E_{w, K}\left[\left(f \circ \tau_{k, k+1}-f\right)^{2}\right] .
\end{aligned}
$$


Notice that $E_{w, K}\left[f \circ \tau_{k, k+1} \mid \mathscr{F}_{k, k+1}\right]=E_{w, K}\left[f \mid \mathscr{F}_{k, k+1}\right]$, so that

$$
E_{w, K}\left[\left(f \circ \tau_{k, k+1}-E_{w, K}\left[f \mid \mathscr{F}_{k, k+1}\right]\right)^{2}\right]=E_{w, K}\left[\left(f-E_{w, K}\left[f \mid \mathscr{F}_{k, k+1}\right]\right)^{2}\right] .
$$

This together with the convex inequality $(a+b)^{2} \leq 2\left(a^{2}+b^{2}\right)$ yields that

$$
E_{w, K}\left[f_{1}\right] \leq 4 K \sum_{k=i}^{j-2} E_{w, K}\left[\left(f-E_{w, K}\left[f \mid \mathscr{F}_{k, k+1}\right]\right)^{2}\right] .
$$

For $f_{2}$, by applying the change of variable $\mathbf{y}=\sigma_{i, j-1} \mathbf{x}$, we obtain that

$$
E_{w, K}\left[f_{2}\right]=E_{w, K}\left[\frac{1}{J_{y_{j-1}+y_{j}}} \int_{0}^{2 \pi}\left[f\left(\rho_{j-1, j}^{\theta} \mathbf{y}\right)-f(\mathbf{y})\right]^{2} \mathfrak{J}_{\mathbf{y}, i, j}(\theta) d \theta\right] .
$$

Therefore, we can calculate this term as

$$
\begin{aligned}
E_{w, K}\left[f_{2}\right] & =2 E_{w, K}\left[f^{2}\right]-2 E_{w, K}\left[f E_{w, K}\left[f \mid \mathscr{F}_{j-1, j}\right]\right] \\
& =E_{w, K}\left[\left(f-E_{w, K}\left[f \mid \mathscr{F}_{j-1, j}\right]\right)^{2}\right] .
\end{aligned}
$$

For $f_{3}$, the same change of variable yields that

$$
E_{w, K}\left[f_{3}\right]=E_{w, K}\left[E_{w, K}\left[\left(f \circ \tilde{\sigma}_{i, j-1}-f\right)^{2} \mid \mathscr{F}_{j-1, j}\right]\right]=E_{w, K}\left[\left(f \circ \tilde{\sigma}_{i, j-1}-f\right)^{2}\right] .
$$

Since $\tilde{\sigma}_{k, j-1}=\tau_{k, k+1} \circ \tilde{\sigma}_{k+1, j-1}$, by repeating the calculation in $f_{1}$,

$$
E_{w, K}\left[f_{3}\right] \leq 4 K \sum_{k=i}^{j-2} E_{w, K}\left[\left(f-E_{w, K}\left[f \mid \mathscr{F}_{k, k+1}\right]\right)^{2}\right] .
$$

Hence, with some universal constant $C<\infty$ we have

$$
E_{w, K}\left[\left(f-E_{w, K}\left[f \mid \mathscr{F}_{i, j}\right]\right)^{2}\right] \leq C K \sum_{k=i}^{j-1} E_{w, K}\left[\left(f \circ \tau_{k, k+1}-f\right)^{2}\right] .
$$

Lemma 6.3 follows by summing up this estimate with $i$ and $j$.

To show Lemma 6.4, we need the following pre-estimate.

Lemma 6.5. Assume 2.1, then 6.2 holds with constants $C_{K}^{\prime}$ satisfying

$$
C_{K}^{\prime} \leq \frac{C^{\prime}}{K}\left(\frac{\delta_{+}}{\delta_{-}}\right)^{3(K-1)} .
$$

Remark 6.6. In view of Lemma 6.5 the spectral gap in (6.1) also holds without the assumption (3.13). In this case, the constants $C_{K}$ satisfies that

$$
C_{K} \leq C K^{2}\left(\frac{\delta_{+}}{\delta_{-}}\right)^{3(K-1)} .
$$

We first prove Lemma 6.4 from Lemma 6.5. The proof of Lemma 6.5 is put in the end of this section. Consider the bounded operator

$$
\mathcal{L}_{K} f=\frac{1}{K} \sum_{1 \leq i<j \leq K}\left(E_{w, K}\left[f \mid \mathscr{F}_{i, j}\right]-f\right), \quad \forall f \text { s.t. } E_{w, K}\left[f^{2}\right]<\infty .
$$


Let $\lambda_{w, K}$ be the spectral gap of $\mathcal{L}_{K}$ on with respect to $E_{w, K}$ :

$$
\lambda_{w, K} \triangleq \inf \left\{\left\langle f,-\mathcal{L}_{K} f\right\rangle_{w, K} \mid E_{w, K}[f]=0, E_{w, K}\left[f^{2}\right]=1\right\},
$$

and let $\lambda_{K}=\inf \left\{\lambda_{w, K} ; w \in \mathbb{R}^{2} \times \mathbb{R}_{+}\right\}$. Then 6.2 is equivalent to

$$
\inf \left\{\lambda_{K} ; K \geq 3\right\}>0 \text {. }
$$

We prove it through an induction argument, firstly established for $k=3,4$ in 8 .

Lemma 6.7. If $k \lambda_{k} \geq 1$ holds for some $k \geq 3$, then for all $K \geq k$,

$$
\lambda_{K} \geq\left(k \lambda_{k}-1\right)\left(\frac{1}{k-2}-\frac{2}{K(k-2)}\right)+\frac{1}{K} .
$$

In view of 3.13 and Lemma 6.5 . for some fixed $k$ which is large enough,

$$
k \lambda_{k}>\frac{k}{C^{\prime}}\left(\frac{\delta_{-}}{\delta_{+}}\right)^{3 k-3} \geq \frac{k}{C^{\prime}} \frac{1}{(1+\delta)^{3 k-3}} \geq 1,
$$

provided that $\delta>0$ is small enough. Then, with Lemma 6.7 we can show that the sequence $\left\{\lambda_{K} ; K \geq 3\right\}$ is uniformly bounded from below.

Proof of Lemma 6.7. We make use of the equivalent characterization of $\lambda_{w, K}$ that

$$
\lambda_{w, K}=\inf \left\{\frac{\left\langle\mathcal{L}_{K} f, \mathcal{L}_{K} f\right\rangle_{w, K}}{\left\langle f,-\mathcal{L}_{K} f\right\rangle_{w, K}} \mid\left\langle f,-\mathcal{L}_{K} f\right\rangle_{w, K} \neq 0\right\} .
$$

In this proof we denote by $B$ the set of all pairs $b=(i, j)$ such that $1 \leq i<j \leq K$, and write $D_{b} f=E_{w, K}\left[f \mid \mathscr{F}_{b}\right]-f$ for all $b \in B$, then

$$
\begin{gathered}
\left\langle\mathcal{L}_{K} f, \mathcal{L}_{K} f\right\rangle_{w, K}=\frac{1}{K^{2}} \sum_{b, b^{\prime} \in B}\left\langle D_{b} f, D_{b^{\prime}} f\right\rangle_{w, K}, \\
\left\langle f,-\mathcal{L}_{K} f\right\rangle_{w, K}=\frac{1}{K} \sum_{b \in B}\left\langle D_{b} f, D_{b} f\right\rangle_{w, K} .
\end{gathered}
$$

We write $b \sim b^{\prime}$ if two pairs $b$ and $b^{\prime}$ have at least one common point. We also consider all the $k$-particle subsets $T_{k} \subseteq\{1, \ldots, K\}$. Notice that if $b \sim b^{\prime}$ but $b \neq b^{\prime}$, there are $\left(\begin{array}{c}K-3 \\ k-3\end{array}\right)$ different $T_{k}$ 's containing both $b$ and $b^{\prime}$. Hence,

$$
\left(\begin{array}{l}
n-3 \\
k-3
\end{array}\right) \sum_{\substack{b, b^{\prime} \in B \\
b \neq b^{\prime}, b \sim b^{\prime}}}\left\langle D_{b} f, D_{b^{\prime}} f\right\rangle_{w, K}=\sum_{T_{k}} \sum_{\substack{b, b^{\prime} \subseteq T_{k} \\
b \neq b^{\prime}, b \sim b^{\prime}}}\left\langle D_{b} f, D_{b^{\prime}} f\right\rangle_{w, K}
$$

If $b \nsim b^{\prime}$, there are $\left(\begin{array}{c}K-4 \\ k-4\end{array}\right)$ different $T_{k}$ 's contain both $b$ and $b^{\prime}$, while for the case $b=b^{\prime}$ it is $\left(\begin{array}{c}K-2 \\ k-2\end{array}\right)$. Therefore, the right-hand side of the equation above equals to

$$
\sum_{T_{k}} \sum_{b, b^{\prime} \subseteq T_{k}}\left\langle D_{b} f, D_{b^{\prime}} f\right\rangle_{w, K}-\left(\begin{array}{c}
K-4 \\
k-4
\end{array}\right) \sum_{b \nsim b^{\prime}}\left\langle D_{b} f, D_{b^{\prime}} f\right\rangle_{w, K}-\left(\begin{array}{c}
K-2 \\
k-2
\end{array}\right) \sum_{b \in B}\left\langle D_{b} f, D_{b} f\right\rangle_{w, K} .
$$

The definition of $\lambda_{k}$ yields that

$$
\frac{1}{k} \sum_{b, b^{\prime} \subseteq T_{k}}\left\langle D_{b} f, D_{b^{\prime}} f\right\rangle_{w, K} \geq \lambda_{k} \sum_{b \subseteq T_{k}}\left\langle D_{b} f, D_{b} f\right\rangle_{w, K}
$$


And for $b \not b^{\prime},\left\langle D_{b} f, D_{b^{\prime}} f\right\rangle_{w, K}=E_{w, K}\left[\left(D_{b^{\prime}} D_{b} f\right)^{2}\right] \geq 0$. Therefore,

$$
\sum_{\substack{b, b^{\prime} \in B \\ b \neq b^{\prime}, b \sim b^{\prime}}}\left\langle D_{b} f, D_{b^{\prime}} f\right\rangle_{w, K} \geq \frac{\left(k \lambda_{k}-1\right)(K-2)}{k-2} \sum_{b \in B}\left\langle D_{b} f, D_{b} f\right\rangle_{w, K} .
$$

By the condition $k \lambda_{k}>1$, the right-hand side is positive. In conclusion,

$$
\begin{aligned}
\left\langle\mathcal{L}_{K} f, \mathcal{L}_{K} f\right\rangle_{w, K} & \geq \frac{1}{K^{2}} \sum_{b \in B}\left\langle D_{b} f, D_{b} f\right\rangle_{w, K}+\frac{1}{K^{2}} \sum_{b \neq b^{\prime}, b \sim b^{\prime}}\left\langle D_{b} f, D_{b} f\right\rangle_{w, K} \\
& \geq \frac{1}{K^{2}}\left[\frac{\left(k \lambda_{k}-1\right)(K-2)}{k-2}+1\right] \sum_{b \in B}\left\langle D_{b} f, D_{b} f\right\rangle_{w, K} \\
& =\left[\left(k \lambda_{k}-1\right)\left(\frac{1}{k-2}-\frac{2}{K(k-2)}\right)+\frac{1}{K}\right]\left\langle f,-\mathcal{L}_{K} f\right\rangle_{w, K} .
\end{aligned}
$$

Notice that this estimate is independent of the choice of $w$.

Finally, to complete the proof of Proposition 6.1. we are left to show Lemma 6.5. To do this, we make use of the spectral gap bound of Kac walk. For $a \in \mathbb{R}^{2}$ and $R \geq|a|^{2}$, consider the $(2 K-3)$-dimensional sphere

$$
S_{K}(a, R)=\left\{x_{1}, \ldots,\left.x_{K} \in \mathbb{R}^{2}\left|\frac{1}{K} \sum_{k=1}^{K} x_{k}=a, \frac{1}{K} \sum_{k=1}^{K}\right| x_{k}\right|^{2}=R\right\} .
$$

Denote by $\mu_{K}(a, R)$ the uniform measure on $S_{K}(a, R)$. With a little abuse of notations, let $\mathscr{F}_{i, j}=\sigma\left\{x_{k} ; k \neq i, j\right\}$ for $1 \leq i<j \leq K$.

Lemma 6.8. There exists a constant $C$ such that

$$
E_{\mu_{K}(a, R)}\left[\left(f-E_{\mu_{K}(a, R)}[f]\right)^{2}\right] \leq \frac{C}{K} \sum_{1 \leq i<j \leq n} E_{\mu_{K}(a, R)}\left[\left(f-E_{\mu_{K}(a, R)}\left[f \mid \mathscr{F}_{i, j}\right]\right)^{2}\right]
$$

for all $(a, R, K)$ and bounded smooth function $f$.

Lemma 6.8 can be proved by the arguments in [9] and 10 . We here prove Lemma 6.5 by applying a perturbation on the spectral gap in Lemma 6.8

Proof. To begin with, from 2.1 we know that for $r \neq r^{\prime}$ and $K \geq 1$,

$$
\frac{\sqrt{2(K+1)}}{\sqrt{K}} c_{-} \leq \frac{\left|V^{\prime}(r)-V^{\prime}\left(r^{\prime}\right)\right|}{\sqrt{V(r)+K V\left(r^{\prime}\right)-(K+1) V\left(\frac{r+K r^{\prime}}{K+1}\right)}} \leq \frac{\sqrt{2(K+1)}}{\sqrt{K}} c_{+},
$$

where $c_{-}=\delta_{-} / \sqrt{\delta_{+}}$and $c_{+}=\delta_{+} / \sqrt{\delta_{-}}$. For each $K \geq 3$, we construct a bijection $\tau_{K}: \Omega_{K} \rightarrow$ $\Omega_{K}$, satisfying the following two conditions.

(i) For $w=(p, r, e), \tau_{K}\left(\Omega_{w, K}\right)=S_{K}(a, R)$, where $a=(p, r), R=2 e-2 V(r)+r^{2}$;

(ii) The Jacobian matrix $\tau_{K}^{\prime}$ of $\tau_{K}$ satisfies that $c_{-}^{K-1} \leq\left|\operatorname{det}\left(\tau_{K}^{\prime}\right)\right| \leq c_{+}^{K-1}$.

Indeed, given a bounded, measurable, positive function $g$ on $\Omega_{w, K}$, by (i) we know that $\tau_{K}^{-1} g:=$ $g \circ \tau_{K}^{-1}$ defines a function on $S_{K}(a, R)$, and (ii) yields that

$$
c_{0}^{-(K-1)} E_{\mu_{K}(a, R)}\left[\tau_{K}^{-1} g\right] \leq E_{w, K}[g] \leq c_{0}^{K-1} E_{\mu_{K}(a, R)}\left[\tau_{K}^{-1} g\right],
$$


where $c_{0}=c_{+} / c_{-}$. For bounded and smooth function $f$, we can apply the estimate above to $g=\left(f-E_{\mu_{K}(a, R)}\left[\tau_{K}^{-1} f\right]\right)^{2}$ to obtain

$$
E_{w, K}\left[\left(f-E_{w, K}[f]\right)^{2}\right] \leq E_{w, K}[g] \leq c_{0}^{K-1} E_{\mu_{K}(a, R)}\left[\tau_{K}^{-1} g\right] .
$$

On the other hand, take $h_{i, j}=\left(f-E_{w, K}\left[f \mid \mathscr{F}_{i, j}\right]\right)^{2}$ and similarly,

$$
E_{\mu_{K}(a, R)}\left[\left(\tau_{K}^{-1} f-E_{\mu_{K}(a, R)}\left[\tau_{K}^{-1} f \mid \mathscr{F}_{i, j}\right]\right)^{2}\right] \leq E_{\mu_{K}(a, R)}\left[\tau_{K}^{-1} h_{i, j}\right] \leq c_{0}^{K-1} E_{w, K}\left[h_{i, j}\right] .
$$

Substituting $\tau_{K}^{-1} f$ for $f$ in Lemma 6.8, we get

$$
\begin{aligned}
E_{w, K}\left[\left(f-E_{w, K}[f]\right)^{2}\right] & \leq c_{0}^{K-1} E_{\mu_{K}(a, R)}\left[\left(\tau_{K}^{-1} f-E_{\mu_{K}(a, R)}\left[\tau_{K}^{-1} f\right]\right)^{2}\right] \\
& \leq \frac{C c_{0}^{K-1}}{K} \sum_{i<j} E_{\mu_{K}(a, R)}\left[\left(\tau_{K}^{-1} f-E_{\mu_{K}(a, R)}\left[\tau_{K}^{-1} f \mid \mathscr{F}_{i, j}\right]\right)^{2}\right] \\
& \leq \frac{C c_{0}^{2(K-1)}}{K} \sum_{i<j} E_{w, K}\left[\left(f-E_{w, K}\left[f \mid \mathscr{F}_{i, j}\right]\right)^{2}\right] .
\end{aligned}
$$

Since $c_{0}=\left(\delta_{+} / \delta_{-}\right)^{3 / 2}$, Lemma 6.5 then follows.

Now fix $K \geq 3$ and we construct the map $\tau_{K}$. Write $x_{k}=\left(p_{k}, r_{k}\right)$ and define

$$
\alpha_{k}=\frac{1}{k} \sum_{i=1}^{k} r_{i}, \quad \forall 1 \leq k \leq K .
$$

Consider two maps $\zeta, \zeta_{*}: \mathbb{R}^{K} \rightarrow \mathbb{R}^{K}$. The first map $\zeta$ is given by

$$
\zeta\left(r_{1}, \ldots, r_{K}\right)=\left(r_{1}^{\prime}, \ldots, r_{K}^{\prime}\right),
$$

such that $r_{K}^{\prime}=\alpha_{K}$, and for $1 \leq k \leq K-1$,

$$
\left(r_{k}^{\prime}\right)^{2}=\frac{2 k}{k+1}\left(V\left(r_{k+1}\right)+k V\left(\alpha_{k}\right)-(k+1) V\left(\alpha_{k+1}\right)\right),
$$

where the sign of $r_{k}^{\prime}$ is chosen in accordance with $r_{k}-\alpha_{K}$. Meanwhile, $\zeta_{*}$ is given by

$$
\zeta_{*}\left(r_{1}^{\prime}, \ldots, r_{K}^{\prime}\right)=\left(r_{1}^{\prime \prime}, \ldots, r_{K}^{\prime \prime}\right)
$$

such that

$$
r_{k}^{\prime \prime}= \begin{cases}r_{K}^{\prime}-\sum_{i=1}^{K-1} \frac{r_{i}^{\prime}}{i}, & \text { for } k=1, \\ r_{K}^{\prime}+r_{k-1}^{\prime}-\sum_{i=k}^{K-1} \frac{r_{i}^{\prime}}{i}, & \text { for } 2 \leq k \leq K-1, \\ r_{K}^{\prime}+r_{K-1}^{\prime}, & \text { for } k=K .\end{cases}
$$

Denote by $J$ and $J_{*}$ the Jacobian matrices of $\zeta$ and $\zeta_{*}$, respectively. To compute $J$, noticing that $\partial_{r_{i}} r_{k}^{\prime}=\partial_{r_{k}} r_{k}^{\prime}$ for all $i \leq k$, and $\partial_{r_{i}} r_{k}^{\prime}=0$ for all $i>k+1$, we have

$$
J=\left[\begin{array}{ccccc}
\frac{\partial r_{1}^{\prime}}{\partial r_{7}} & \frac{\partial r_{1}^{\prime}}{\partial r_{2}} & 0 & \ldots & 0 \\
\frac{\partial r_{2}}{\partial r_{2}} & \frac{\partial r_{2}^{\prime}}{\partial r_{2}} & \frac{\partial r_{2}^{\prime}}{\partial r_{3}} & \cdots & 0 \\
\vdots & \vdots & \vdots & & \vdots \\
\frac{\partial r_{K-1}^{\prime}}{\partial r_{K}-1} & \frac{\partial r_{K-1}^{\prime}}{\partial r_{K}-1} & \frac{\partial r_{K-1}^{\prime}}{\partial r_{K}-1} & \ldots & \frac{\partial r_{K-1}^{\prime}}{\partial r_{K}} \\
\frac{\partial r_{K}^{\prime}}{\partial r_{K}} & \frac{\partial r_{K}^{\prime}}{\partial r_{K}} & \frac{\partial r_{K}^{\prime}}{\partial r_{K}} & \cdots & \frac{\partial r_{K}^{\prime}}{\partial r_{K}}
\end{array}\right] .
$$


Hence, its determinant reads

$$
|\operatorname{det}(J)|=\left|\frac{\partial r_{K}^{\prime}}{\partial r_{K}}\right| \cdot \prod_{k=1}^{K-1}\left|\frac{\partial r_{k}^{\prime}}{\partial r_{k}}-\frac{\partial r_{k}^{\prime}}{\partial r_{k+1}}\right|
$$

Since $\partial_{r_{K}} r_{K}^{\prime}=1 / K$ and for $k=1, \ldots, K-1$ we have

$$
\frac{\partial r_{k}^{\prime}}{\partial r_{i}}= \begin{cases}\frac{k}{(k+1) r_{k}^{\prime}}\left[V^{\prime}\left(\alpha_{k}\right)-V^{\prime}\left(\alpha_{k+1}\right)\right], & \text { if } 1 \leq i \leq k \\ \frac{k}{(k+1) r_{k}^{\prime}}\left[V^{\prime}\left(r_{k+1}\right)-V^{\prime}\left(\alpha_{k+1}\right)\right], & \text { if } i=k+1\end{cases}
$$

In consequence, $|\operatorname{det}(J)|$ equals to

$$
\frac{1}{K} \prod_{k=1}^{K-1} \frac{\sqrt{k}}{\sqrt{2(k+1)}} \frac{\left|V^{\prime}\left(r_{k+1}\right)-V^{\prime}\left(\alpha_{k}\right)\right|}{\sqrt{V\left(r_{k+1}\right)+k V\left(\alpha_{k}\right)-(k+1) V\left(\alpha_{k+1}\right)}} .
$$

Applying the estimate in (6.4) to obtain that

$$
\frac{c_{-}^{K-1}}{K} \leq|\operatorname{det}(J)| \leq \frac{c_{+}^{K-1}}{K} .
$$

Meanwhile it is easy to calculate that $\left|\operatorname{det}\left(J_{*}\right)\right|=K$. Therefore, define

$$
\tau_{K}:\left(p_{1}, \ldots, p_{K}, r_{1}, \ldots, r_{K}\right) \mapsto\left(p_{1}, \ldots, p_{K}, r_{1}^{\prime \prime}, \ldots, r_{K}^{\prime \prime}\right),
$$

then $\left|\operatorname{det}\left(\tau_{K}^{\prime}\right)\right|$ satisfies (ii). On the other hand, suppose that $\left\{x_{k}=\left(p_{k}, r_{k}\right)\right\}_{1 \leq k \leq K}$ belongs to the microcanonical manifold $\Omega_{w, K}$, then $r_{K}^{\prime}=r$ and

$$
\frac{1}{K} \sum_{k=1}^{K-1} \frac{k+1}{k} \frac{\left(r_{k}^{\prime}\right)^{2}}{2}=\frac{1}{K} \sum_{k=1}^{K} V\left(r_{k}\right)-V\left(r_{K}^{\prime}\right)=e-\frac{1}{K} \sum_{k=1}^{K} \frac{p_{k}^{2}}{2}-V(r) .
$$

Then, it follows from the definition of $r_{k}^{\prime \prime}$ that

$$
\frac{1}{K} \sum_{k=1}^{K}\left(r_{k}^{\prime \prime}\right)^{2}=\left(r_{K}^{\prime}\right)^{2}+\frac{1}{K} \sum_{k=1}^{K-1} \frac{k+1}{k}\left(r_{k}^{\prime}\right)^{2}=2 e-2 V(r)+r^{2}-\frac{1}{K} \sum_{k=1}^{K} p_{k}^{2}
$$

Hence, $\tau_{K}\left(x_{1}, \ldots, x_{K}\right) \in S_{K}(a, R)$ with $R=2 e-V(r)+r^{2}$, and (i) is also verified. The proof of Lemma 6.5 is then completed.

\section{Tightness}

In Section 4 we have proved the convergence of the finite-dimensional distribution of $\left\{\mathbb{Q}_{N}\right\}$. In order to complete the proof of Theorem 3.1, we need its tightness in $C\left([0, T], \mathscr{H}_{-k}(\lambda)\right)$. The proof is standard, and we summarize it here.

It suffices to show the two statements below:

$$
\begin{aligned}
& \lim _{M \rightarrow \infty} \limsup _{N \rightarrow \infty} \mathbb{P}_{\lambda, N}\left\{\sup _{t \in[0, T]}\left\|Y_{N}(t)\right\|_{-k} \geq M\right\}=0, \\
& \lim _{\delta \downarrow 0} \limsup _{N \rightarrow \infty} \mathbb{P}_{\lambda, N}\left\{w_{-k}\left(Y_{N}, \delta\right) \geq \epsilon\right\}=0, \quad \forall \epsilon>0,
\end{aligned}
$$

where $w_{-k}\left(Y_{N}, \delta\right)$ is the modulus of continuity in $C\left([0, T], \mathscr{H}_{-k}(\lambda)\right)$. Recall that

$$
\left\|Y_{N}\right\|_{-k}^{2}=\sum_{i=1}^{2} \sum_{n=0}^{\infty}\left\{\theta_{n}^{-2 k} Y_{N}^{2}\left(R \boldsymbol{\mu}_{i, n}\right)+\kappa_{n}^{-2 k} Y_{N}^{2}\left(R \boldsymbol{\nu}_{i, n}\right)\right\},
$$


where $R$ is the rotation matrix in $(3.9)$, and $\boldsymbol{\mu}_{i, n}, \boldsymbol{\nu}_{i, n}$ are the three-dimensional Fourier bases defined in (3.4) and (3.8).

Take $f=\boldsymbol{\mu}_{i, n}$ or $\boldsymbol{\nu}_{i, n}$ for some $(i, n)$. Applying (4.1) with $H(t) \equiv R f$,

$$
Y_{N}(t, R f)=Y_{0}(0, R f)+\int_{0}^{t} Y_{N}\left(s, L^{*}[R f]\right) d s+\epsilon_{N}(t, f),
$$

and by Lemma 4.1 and Remark $4.3, \epsilon_{N}$ satisfies that

$$
\mathbb{E}_{\lambda, N}\left[\sup _{t \in[0, T]} \epsilon_{N}^{2}(t, f)\right]=o_{N}(1)\left(\left|f^{\prime}\right|_{\infty}^{2}+\left|f^{\prime \prime}\right|_{\infty}^{2}+\left\|f^{\prime}\right\|^{2}\right) .
$$

On the other hand, it is easy to see that

$$
\mathbb{E}_{\lambda, N}\left[\sup _{t \in[0, T]}\left|\int_{0}^{t} Y\left(s, L^{*}[R f]\right) d s\right|^{2}\right] \leq C T^{2}\left\|f^{\prime}\right\|^{2} .
$$

Observe that $\left|f_{i, n}^{\prime \prime}(x)\right| \leq \sqrt{2} \max \left\{\theta_{n}^{2}, \kappa_{n}^{2}\right\}$. Then, for $k>5 / 2$, 7.1) and 7.2 can be proved by standard arguments (cf. [13, 11.3]).

\section{Equivalence of ensembles}

In this section we prove the equivalence of ensembles for the dynamics with multi-dimensional conserved quantities. By applying Proposition 8.3 to the the model in this paper, we obtain Corollary 8.4, which is necessary in the proof of Lemma 4.2 .

The notations in this section are different from the former part. Let $\pi$ be a Borel measure on $\Omega=\mathbb{R}^{m}$ with smooth density function with respect to the Lebesgue measure, and $\mathbf{f}=$ $\left(f_{1}, \ldots, f_{d}\right)$ be a $d$-dimensional function on $\Omega$ with compact level sets. Suppose that there is some domain $D \subseteq \mathbb{R}^{d}$, such that

$$
Z(\lambda) \triangleq \log \left[\int_{\Omega} \exp \{\lambda \cdot \mathbf{f}(\omega)\} \pi(d \omega)\right]<\infty, \quad \forall \lambda \in D .
$$

To avoid the problem of regularity, we assume that $Z$ is four times continuously differentiable on $D$, and its Hessian matrix $\Sigma_{\lambda}=Z^{\prime \prime}(\lambda)$ is always positive-definite. To simplify the notations we denote $\mathbf{u}_{\lambda}=\nabla_{\lambda} Z(\lambda)$.

For $\lambda \in D$ we can define the tilted probability measure by

$$
\pi_{\lambda}(d \omega) \triangleq \exp \{\lambda \cdot \mathbf{f}(\omega)-Z(\lambda)\} \pi(d \omega) .
$$

Observe that $E_{\pi_{\lambda}}[\mathbf{f}]=\mathbf{u}_{\lambda}$, and $E_{\pi_{\lambda}}\left[\left(\mathbf{f}-\mathbf{u}_{\lambda}\right)\left(\mathbf{f}-\mathbf{u}_{\lambda}\right)^{\prime}\right]=\Sigma_{\lambda}$. Let $\Phi_{\lambda}$ be the centered characteristic function of $\mathbf{f}$ with respect to $\pi_{\lambda}$, given by

$$
\Phi_{\lambda}(\mathbf{h})=\int_{\Omega} \exp \left\{i \mathbf{h} \cdot\left(\mathbf{f}(\omega)-\mathbf{u}_{\lambda}\right)\right\} \pi_{\lambda}(d \omega), \quad \forall \mathbf{h} \in \mathbb{R}^{d} .
$$

We also assume that there exists some $\epsilon_{0}>0$, such that

$$
\sup _{\mathbf{h} \in \mathbb{R}^{d}}|\mathbf{h}|^{\epsilon_{0}}\left|\Phi_{\lambda}(\mathbf{h})\right|<\infty .
$$

The main methods we use here is a multi-dimensional local central limit theorem with an edge expansion and a large deviation property for $\mathbf{f}$. We state them in Lemma 8.1 and Lemma 8.2 respectively. Let $\phi_{\lambda}=\phi_{\lambda}(\mathbf{x})$ be the Gaussian density function on $\mathbb{R}^{d}$, whose mean is $\mathbf{0}$ and variance matrix is $\Sigma_{\lambda}$ :

$$
\phi_{\lambda}(\mathbf{x})=\frac{1}{(2 \pi)^{d / 2}} \frac{1}{\sqrt{\operatorname{det} \Sigma_{\lambda}}} \exp \left\{-\frac{\mathbf{x}^{\prime} \Sigma_{\lambda}^{-1} \mathbf{x}}{2}\right\}, \quad \forall \mathbf{x} \in \mathbb{R}^{d} .
$$


For $k \in \mathbb{N}_{+}$, define the $d$-varibale polynomials $P_{\lambda, k}$ by

$$
P_{\lambda, k}(\mathbf{h})=\sum_{|\alpha|=k} \frac{\partial_{\alpha} Z(\lambda)}{\alpha !} \mathbf{h}^{\alpha},
$$

where $\alpha=\left(\alpha_{1}, \ldots, \alpha_{d}\right)$ is multiple index, $\alpha_{j} \geq 0$, and

$$
|\alpha|=\sum_{j=1}^{d} \alpha_{j}, \quad \alpha !=\prod_{j=1}^{d} \alpha_{j} !, \quad \partial_{\alpha}=\prod_{j=1}^{d} \frac{\partial^{\alpha_{j}}}{\partial \lambda_{j}^{\alpha_{j}}}, \quad \mathbf{h}^{\alpha}=\prod_{j=1}^{d} h_{j}^{\alpha_{j}} .
$$

Also define the polynomials $Q_{\lambda, 3}$ and $Q_{\lambda, 4}$ by

$$
\begin{gathered}
Q_{\lambda, 3}=\frac{1}{(2 \pi)^{d} \phi_{\lambda}} \int_{\mathbb{R}^{d}} \exp \left\{-i \mathbf{x} \cdot \mathbf{h}-\frac{\mathbf{h}^{\prime} \Sigma_{\lambda} \mathbf{h}}{2}\right\} P_{\lambda, 3}(i \mathbf{h}) d \mathbf{h} ; \\
Q_{\lambda, 4}=\frac{1}{(2 \pi)^{d} \phi_{\lambda}} \int_{\mathbb{R}^{d}} \exp \left\{-i \mathbf{x} \cdot \mathbf{h}-\frac{\mathbf{h}^{\prime} \Sigma_{\lambda} \mathbf{h}}{2}\right\}\left(P_{\lambda, 4}+\frac{P_{\lambda, 3}^{2}}{2}\right)(i \mathbf{h}) d \mathbf{h} .
\end{gathered}
$$

Let $\Omega_{n}$ be the $n$-product space of $\Omega$. Define

$$
\mathbf{f}_{(n)}(\vec{\omega})=\frac{1}{n} \sum_{j=1}^{n} \mathbf{f}\left(\omega_{j}\right), \quad \forall \vec{\omega}=\left(\omega_{1}, \ldots, \omega_{n}\right) \in \Omega_{n}
$$

Equip $\Omega_{n}$ with the product measure $\pi_{\lambda, n}=\otimes_{j} \pi_{\lambda}\left(d \omega_{j}\right)$. We have the following local central limit theorem. The proof is standard [19, Theorem VII.15].

Lemma 8.1. Let $f_{\lambda, n}$ be the density function of the random vector

$$
\sqrt{n}\left(\mathbf{f}_{(n)}-\mathbf{u}_{\lambda}\right)=\frac{1}{\sqrt{n}} \sum_{j=1}^{n}\left(\mathbf{f}\left(\omega_{j}\right)-\mathbf{u}_{\lambda}\right)
$$

with respect to the product measure $\pi_{\lambda, n}$ for $n$ large enough. Then,

$$
\left|f_{\lambda, n}(\mathbf{x})-\phi_{\lambda}(\mathbf{x})\left(1+\frac{Q_{\lambda, 3}(\mathbf{x})}{\sqrt{n}}+\frac{Q_{\lambda, 4}(\mathbf{x})}{n}\right)\right| \leq \frac{K_{\lambda, n}}{n}, \quad \forall \mathbf{x} \in \mathbb{R}^{d},
$$

where $\lim _{n \rightarrow \infty} K_{\lambda, n}=0$, uniformly in any compact subset of $D$.

As $Z$ is strictly convex, consider its Fenchel-Legendre transform:

$$
Z^{*}(\mathbf{u})=\sup _{\lambda \in D}\{\lambda \cdot \mathbf{u}-Z(\lambda)\}
$$

Let $D^{*}=\left\{\mathbf{u} \in \mathbb{R}^{d}: Z^{*}(\mathbf{u})<\infty\right\}$. The superior is reached at a unique $\lambda(\mathbf{u}) \in D$, given by the convex conjugate

$$
\lambda(\mathbf{u})=\nabla_{\mathbf{u}} Z^{*}(\mathbf{u}), \quad \mathbf{u}_{\lambda}=\nabla_{\lambda} Z(\lambda) .
$$

Notice that $\mathbf{u} \mapsto \lambda(\mathbf{u})$ and $\lambda \mapsto \mathbf{u}_{\lambda}$ are a pair of inverse maps between $D$ and $D^{*}$. For $\lambda \in D$ and $\mathbf{u} \in D^{*}$, define the rate function $I_{\lambda}(\mathbf{u})$ by

$$
I_{\lambda}(\mathbf{u})=Z^{*}(\mathbf{u})-Z^{*}\left(\mathbf{u}_{\lambda}\right)-\nabla_{\mathbf{u}} Z^{*}\left(\mathbf{u}_{\lambda}\right) \cdot\left(\mathbf{u}-\mathbf{u}_{\lambda}\right) .
$$

Denote by $M_{\lambda}$ the largest eigenvalue of $\Sigma_{\lambda}$. By the arguments above it is not hard to conclude that for any constant $M>M_{\lambda}$, we have

$$
I_{\lambda}(\mathbf{u}) \geq(2 M)^{-1}\left|\mathbf{u}-\mathbf{u}_{\lambda}\right|^{2}
$$

holds if $\left|\mathbf{u}-\mathbf{u}_{\lambda}\right|$ is small enough. By virtue of (8.3), we can also obtain the following large deviation property for $\mathbf{f}_{(n)}$. 
Lemma 8.2. For any $M>M_{\lambda}$, there exists some $\delta_{M}$ such that

$$
\pi_{\lambda, n}\left\{\left|\mathbf{f}_{(n)}-\mathbf{u}_{\lambda}\right| \geq \delta\right\} \leq 2^{d} \exp \left(-\frac{n M \delta^{2}}{d}\right),
$$

holds for all $n \geq 1$ when $|\delta|<\delta_{M}$.

Proof. Let $\Gamma \subseteq \mathbb{R}^{d}$ be the collection of vectors whose coordinates are all \pm 1 . Notice that the following inequality holds for all $\mathbf{x} \in \mathbb{R}^{d}$ :

$$
e^{|\mathbf{x}|} \leq \prod_{j=1}^{d} e^{\left|x_{j}\right|} \leq \prod_{j=1}^{d}\left(e^{-x_{j}}+e^{x_{j}}\right)=\sum_{\gamma \in \Gamma} e^{\gamma \cdot \mathbf{x}} .
$$

By exponential Chebyshev's inequality and the above estimate, for $\theta>0$,

$$
\begin{aligned}
\pi_{\lambda, n}\left\{\left|\mathbf{f}_{(n)}-\mathbf{u}_{\lambda}\right| \geq \delta\right\} & \leq \sum_{\gamma \in \Gamma} e^{-n \theta \delta} \int_{\left|\mathbf{f}_{(n)}-\mathbf{u}_{\lambda}\right| \geq \delta} \exp \left\{n \theta \gamma \cdot\left(\mathbf{f}_{(n)}-\mathbf{u}_{\lambda}\right)\right\} \pi_{\lambda, n}(d \vec{\omega}) \\
& \leq \sum_{\gamma \in \Gamma} \exp \left\{-n \theta u^{\prime}+n Z(\lambda+\theta \gamma)-n Z(\lambda)\right\},
\end{aligned}
$$

where $u^{\prime}=\gamma \cdot \mathbf{u}_{\lambda}+\delta$. To optimize this estimate, define

$$
I_{\lambda, \gamma}\left(u^{\prime}\right)=\sup _{\theta>0}\left\{\theta u^{\prime}-Z(\lambda+\theta \gamma)+Z(\lambda)\right\}=\sup _{\theta \in \mathbb{R}}\left\{\theta u^{\prime}-Z(\lambda+\theta \gamma)+Z(\lambda)\right\}
$$

The last equality is due to the fact that $u^{\prime}-\left.\partial_{\theta} Z(\lambda+\theta \gamma)\right|_{\theta=0}=\delta>0$. Notice that $I_{\lambda, \gamma}$ is the rate function defined in 8.2 corresponding to the measure $\pi_{\lambda}$ and the function $\gamma \cdot \mathbf{f}$. By the arguments which has been used to derive $(8.3)$, one obtains that $I_{\lambda, \gamma}\left(u^{\prime}\right) \geq M_{\lambda}|\gamma|^{-2} \delta^{2}$. The estimate in Lemma 8.2 then follows directly.

Now fix some $k \in \mathbb{N}_{+}$. For an integrable function $G$ on $\Omega_{k}$, any $n \geq k$ and $\mathbf{u} \in D^{*}$, define the microcanonical expectation $\langle G \mid \mathbf{u}\rangle_{n}$ by

$$
\langle G \mid \mathbf{u}\rangle_{n}=E_{\pi_{\lambda, n}}\left[G \mid \mathbf{f}_{(n)}=\mathbf{u}\right] .
$$

It is easy to see that the definition of $\langle G \mid \mathbf{u}\rangle_{n}$ does not depend on $\lambda$. Notice that though the conditional expectation can usually be defined only in a almost sure sense, under the regularity of $\mathbf{f}$, the microcanonical surface

$$
\Omega_{\mathbf{u}, n}=\left\{\vec{\omega} \in \Omega_{n} ; \mathbf{f}_{(n)}(\vec{\omega})=\mathbf{u}\right\}
$$

is smooth enough to define the regular conditional expectation for everywhere in $D^{*}$. Recall that $\mathbf{u}_{\lambda}=E_{\pi_{\lambda}}[\mathbf{f}]$. The following estimate (cf. [13, p.353, Corollary A2.1.4]) holds.

Proposition 8.3. Suppose that for some compact subset $D_{0}$ of $D$,

$$
C_{j} \triangleq \sup _{\lambda \in D_{0}} E_{\pi_{\lambda}}\left[\left|\mathbf{f}-\mathbf{u}_{\lambda}\right|^{j}\right]<\infty, \quad j=1,2,3,4,
$$

and $G: \Omega_{k} \rightarrow \mathbb{R}$ satisfies that $E_{\pi_{\lambda, k}}\left[G^{2}\right]<\infty$ for all $\lambda \in D_{0}$. Then,

$$
\limsup _{n \rightarrow \infty} n\left|\left\langle G \mid \mathbf{u}_{\lambda}\right\rangle_{n}-E_{\pi_{\lambda, k}}[G]\right| \leq C k \sqrt{E_{\pi_{\lambda, k}}\left[\left(G-E_{\pi_{\lambda, k}}[G]\right)^{2}\right]}
$$

with a uniform constant $C$ for every $\lambda \in D_{0}$. 
Proof. The proof is exactly parallel to [13, Corollary A2.1.4]. We sketch it for completeness. Without loss of generality we can assume that $E_{\pi_{\lambda, k}}[G]=0$ for some fixed $\lambda \in D_{0}$. Denote by $F_{\lambda, n}$ the density function of $\mathbf{f}_{(n)}$ under $\pi_{\lambda, n}$ :

$$
\int_{\Omega_{n}} g\left(\mathbf{f}_{(n)}\right) d \pi_{\lambda, n}=\int_{\mathbb{R}^{d}} g(\mathbf{u}) F_{\lambda, n}(\mathbf{u}) d \mathbf{u}
$$

for all integrable function $g$ on $\mathbb{R}^{d}$. We can write $\langle G \mid \mathbf{u}\rangle_{n}$ as

$$
\int_{\mathbb{R}^{k}} G\left(\omega_{1}, \ldots, \omega_{k}\right)\left(\frac{F_{\lambda, n-k}\left(\mathbf{u}_{k, n}\right)}{F_{\lambda, n}(\mathbf{u})}-1\right) \pi_{\lambda, k}\left(d \omega_{1} \ldots d \omega_{k}\right),
$$

where $\mathbf{u}_{k, n}=(n-k)^{-1}\left(n \mathbf{u}-k \mathbf{f}_{(k)}\right)$. Schwarz inequality then yields that

$$
\langle G \mid \mathbf{u}\rangle_{n}^{2} \leq E_{\pi_{\lambda, k}}\left[G^{2}\right] E_{\pi_{\lambda, k}}\left[\left|\frac{F_{\lambda, n-k}\left(\mathbf{u}_{k, n}\right)}{F_{\lambda, n}(\mathbf{u})}-1\right|^{2}\right] .
$$

Take $\mathbf{u}=\mathbf{u}_{\lambda}$ in the above expression. By Lemma 8.1

$$
\left|\frac{F_{\lambda, n-k}\left(\mathbf{u}_{k, n}\right)}{F_{\lambda, n}\left(\mathbf{u}_{\lambda}\right)}-1\right| \leq \frac{C k}{n}\left(1+\left|\mathbf{f}_{(k)}-\mathbf{u}_{\lambda}\right|+k\left|\mathbf{f}_{(k)}-\mathbf{u}_{\lambda}\right|^{2}\right) .
$$

where $C$ is a constant depending on $\left\{C_{j} ; j=1,2,3,4\right\}$, the polynomials $Q_{\lambda, 3}, Q_{\lambda, 4}$ and the sequence $K_{\lambda, n}$ appeared in 8.1. Hence, 8.4 holds for the fixed $\lambda$ we chosen. Since the polynomials $Q_{\lambda, 3}$ and $Q_{\lambda, 4}$ are continuously dependent on $\lambda$, and $K_{\lambda, n}$ vanishes uniformly in $D_{0}$, we can extend the result to every $\lambda \in D_{0}$.

Now we apply Proposition 8.3 to the model established in Section 1 Let $\Omega=\mathbb{R}^{2}, \pi$ be the Lebesgue measure, and $\mathbf{f}$ be the three-dimensional function on $\Omega$ given by

$$
\mathbf{f}(\omega)=\left(p, r,-p^{2} / 2-V(r)\right), \quad \text { for } \omega=(p, r) \in \Omega,
$$

where $V$ is a $C^{4}$-smooth function with quadratic growth 2.1). It is not hard to obtain that $D=\mathbb{R}^{2} \times \mathbb{R}_{+}$and $D^{*}=\mathbb{R}^{2} \times \mathbb{R}_{-}$. For $\lambda \in D$,

$$
Z(\lambda)=\ln \left(\int_{\mathbb{R}} e^{-\lambda_{3} V(r)+\lambda_{2} r} d r\right)+\frac{\lambda_{1}^{2}}{2 \lambda_{3}}+\frac{1}{2} \ln \left(\frac{2 \pi}{\lambda_{3}}\right), \quad \lambda=\left(\lambda_{1}, \lambda_{2}, \lambda_{3}\right) .
$$

So $Z$ is four times differentiable and all of its partial derivatives are uniformly bounded in $[-K, K]^{2} \times[\epsilon, \infty)$ for $K, \epsilon>0$. Furthermore, the assumptions in Proposition 8.3 holds in the same set. Recall the continuous map $\mathbf{u} \rightarrow \lambda(\mathbf{u})$ form $D^{*}$ and $D$, which gives the inverse of $\lambda \rightarrow \mathbf{u}_{\lambda}$. With Proposition 8.3 , we have the following estimate.

Corollary 8.4. Suppose that $F$ is a function on $\Omega_{k}$, such that $E_{\pi_{\lambda, k}}[F]$ is twice continuously differentiable in $\lambda$, and $E_{\pi_{\lambda^{\prime}, k}}\left[F^{4}\right]<\infty$ for some fixed $\lambda^{\prime} \in D$. Define

$$
G=F-E_{\pi_{\lambda^{\prime}, k}}[F]-\left.\nabla_{\mathbf{u}} E_{\pi_{\lambda(\mathbf{u}), k}}\right|_{\mathbf{u}=\mathbf{u}^{\prime}} \cdot\left(\mathbf{f}\left(\omega_{j}\right)-\mathbf{u}^{\prime}\right),
$$

where $\mathbf{u}^{\prime}=\mathbf{u}_{\lambda^{\prime}} \in D^{*}$. Then for $n$ large enough, we have

$$
E_{\lambda^{\prime}, n}\left[\langle G \mid \mathbf{u}\rangle_{n}^{2}\right] \leq C n^{-2},
$$

where $C$ is a finite constant depending only on $F$ and $\lambda^{\prime}$. 
Proof. Fix some $\delta \in\left(0, \delta_{M}\right)$, where $\delta_{M}$ is the constant appeared in Lemma 8.2 . By Schwarz inequality and Lemma 8.2 ,

$$
E_{\lambda^{\prime}, n}\left[\langle G \mid \mathbf{u}\rangle_{n}^{2} \mathbf{1}_{\left\{\left|\mathbf{u}-\mathbf{u}^{\prime}\right|>\delta\right\}}\right] \leq 2^{\frac{d}{2}} \exp \left\{-\frac{n M \delta^{2}}{2 d}\right\} \sqrt{E_{\lambda^{\prime}, n}\left[\langle G \mid \mathbf{u}\rangle_{n}^{4}\right]},
$$

so it suffices only consider the compact set $\left\{\left|\mathbf{u}-\mathbf{u}^{\prime}\right| \leq \delta\right\}$. Observe that

$$
\langle G \mid \mathbf{u}\rangle_{n}=\langle F \mid \mathbf{u}\rangle_{n}-E_{\pi_{\lambda^{\prime}, k}}[F]-\left.\nabla_{\mathbf{u}} E_{\pi_{\lambda(\mathbf{u}), k}}\right|_{\mathbf{u}=\mathbf{u}^{\prime}} \cdot\left(\mathbf{u}-\mathbf{u}^{\prime}\right), \quad \forall \mathbf{u} \in D^{*} .
$$

Recall that $\lambda(\mathbf{u})$ continuous in $D^{*}$, so $\left\{\lambda(\mathbf{u}) ;\left|\mathbf{u}-\mathbf{u}^{\prime}\right| \leq \delta\right\}$ is a compact subset of $D$. Apply Proposition 8.3 with $\lambda=\lambda(\mathbf{u})$ to obtain that

$$
\left|\langle F \mid \mathbf{u}\rangle_{n}-E_{\pi_{\lambda(\mathbf{u}), k}}[F]\right| \leq C n^{-1}, \quad \forall \mathbf{u} \in\left\{\left|\mathbf{u}-\mathbf{u}^{\prime}\right| \leq \delta\right\},
$$

where the constant $C=C(F, \delta)$, so its square integral is bounded by $C^{\prime} n^{-2}$. We are left with the second moment in $\left\{\left|\mathbf{u}-\mathbf{u}^{\prime}\right| \leq \delta\right\}$ of

$$
E_{\pi_{\lambda(\mathbf{u}), k}}[F]-E_{\pi_{\lambda^{\prime}, k}}[F]-\left.\nabla_{\mathbf{u}} E_{\pi_{\lambda(\mathbf{u}), k}}\right|_{\mathbf{u}=\mathbf{u}^{\prime}} \cdot\left(\mathbf{u}-\mathbf{u}^{\prime}\right) .
$$

Since $E_{\pi_{\lambda, n}}[F]$ is smooth in $\lambda$ and $\lambda(\mathbf{u})$ is smooth in $\mathbf{u}$, we know that this function is bounded by $C\left|\mathbf{u}-\mathbf{u}_{\lambda}\right|$, with some constant $C=C\left(F, \lambda^{\prime}\right)$. The desired estimate then follows from the fact that $E_{\lambda^{\prime}, n}\left[\left|\mathbf{f}_{(n)}-\mathbf{u}^{\prime}\right|^{4}\right] \leq C^{\prime} n^{-2}$.

\section{Acknowledgments}

This work has been partially supported by the grants ANR-15-CE40-0020-01 LSD of the French National Research Agency. We thank Makiko Sasada for the insightful discussion about the spectral gap estimate (cf. Section 6).

\section{References}

[1] Giada Basile, Cédric Bernardin, Milton Jara, Tomasz Komorowski, and Stefano Olla. Thermal conductivity in harmonic lattices with random collisions. In Stefano Lepri, editor, Thermal transport in low dimensions: from statistical physics to nanoscale heat transfer, volume 921 of Lecture Notes in Physics, chapter 5. Springer, 2016.

[2] Cédric Bernardin, Patricia Gonçalves, and Milton Jara. 3/4-fractional superdiffusion in a system of Harmonic oscillators perturbed by a conservative noise. Arch. Rational Mech. Anal., 220(2):505-542, 2016.

[3] Cédric Bernardin, Patricia Gonçalves, Milton Jara, Makiko Sasada, and Marielle Simon. From normal diffusion to superdiffusion of energy in the evanescent flip noise limit. J. Stat. Phys., 159(6):1327-1368, 2015.

[4] Cédric Bernardin, Patricia Gonçalves, Milton Jara, and Marielle Simon. Nonlinear perturbation of a noisy Hamiltonian lattice field model: universality persistence. Comm. Math. Phys., 361(2):605-659, 2018.

[5] Cédric Bernardin, Francois Huveneers, and Stefano Olla. Hydrodynamic limit for a disordered harmonic chain. Comm. Math. Phys., 2018.

[6] Nadine Braxmeier-Even and Stefano Olla. Hydrodynamic limit for an Hamiltonian system with boundary conditions and conservative noise. Arch. Ration. Mech. Appl., 213(2):561$585,2014$. 
[7] Thomas M. Brox and Hermann Rost. Equilibrium fluctuations of stochastic particle systems: the role of conserved quantities. Ann. Probab., 12(3):742-759, 1984.

[8] Pietro Caputo. On the spectral gap of the Kac walk and other binary collision processes. ALEA - Latin American J. Probab. Math. Stat., 4:205-222, 2008.

[9] Eric A. Carlen, Maria C. Carvalho, and Michael Loss. Determination of the spectral gap for Kac's master equation and related stochastic evolution. Acta Math., 191(1):1-54, 2003.

[10] Eric A. Carlen, Jeffrey S. Geronimo, and Michael Loss. Determination of the spectral gap in the Kac model for physical momentum and energy-conserving collisions. SIAM J. Math. Anal., 40(1):327-364, 2008.

[11] József Fritz, Tadahisa Funaki, and Joel L. Lebowitz. Stationary states of random Hamiltonian systems. Probab. Theory Relat. Fields, 99(2):211-236, 1994.

[12] Milton Jara, Tomasz Komorowski, and Stefano Olla. Superdiffusion of energy in a chain of harmonic oscillators with noise. Comm. Math. Phys., 339(2):407-453, 2015.

[13] Claude Kipnis and Claudio Landim. Scaling limits of interacting particle systems, volume 320 of Grundlehren der mathematischen wissenschaften. Springer-Verlag Berlin Heidelberg, 1999.

[14] Tomasz Komorowski, Claudio Landim, and Stefano Olla. Fluctuations in Markov processes. Time symmetry and martingale approximation, volume 345 of Grundlehren der Mathematischen Wissenschaften. Springer-Verlag Heidelberg, 2012.

[15] Tomasz Komorowski and Stefano Olla. Ballistic and superdiffusive scales in the macroscopic evolution of a chain of oscillators. Nonlinearity, 29(3):962-999, 2016.

[16] Charles B. Morrey. On the derivation of the equations of hydrodynamics from statistical mechanics. Comm. Pure Appl. Math., 8(2):279-326, 1955.

[17] Stefano Olla and Makiko Sasada. Macroscopic energy diffusion for a chain of anharmonic oscillators. Probab. Theory Relat. Fields, 157(3-4):721-775, 2013.

[18] Stefano Olla, Srinivasa R. S. Varadhan, and Horng-Tzer Yau. Hydrodynamical limit for a Hamiltonian system with weak noise. Comm. Math. Phys., 155(3):523-560, 1993.

[19] Valentin V. Petrov. Sums of Independent Random Variables. Translated form the Russian original edition by Arthur A. Brown, volume 82 of Ergebnisse der Mathematik und ihrer Grenzgebiete. Springer-Verlag Berlin Heidelberg New York, 1975.

[20] Fraydoun Rezakhanlou. Microscopic structure of shocks in one conservation laws. Ann. Inst. Henri Poincaré Nonlinear Anal., 12(2):119-153, 1995.

[21] Herbert Spohn. Nonlinear fluctuating hydrodynamics for anharmonic chains. J. Stat. Phys., 154(5):1191-1227, 2014.

Stefano Olla

CEREMADE, UMR-CNRS, Université de Paris Dauphine, PSL Research University

Place du Maréchal De Lattre De Tassigny, 75016 Paris, France

olla@ceremade.dauphine.fr

$\mathrm{Lu} \mathrm{Xu}$

CEREMADE, UMR-CNRS, Université de Paris Dauphine, PSL Research University

Place du Maréchal De Lattre De Tassigny, 75016 Paris, France

xu@ceremade.dauphine.fr 